\title{
Feasibility Studies for Quarkonium Production at a Fixed-Target Experiment Using the LHC Proton and Lead Beams (AFTER@LHC)
}

\author{
L. Massacrier, ${ }^{1,2}$ B. Trzeciak, ${ }^{3}$ F. Fleuret, ${ }^{4}$ C. Hadjidakis, ${ }^{2}$ D. Kikola, ${ }^{5}$ \\ J. P. Lansberg, ${ }^{2}$ and H.-S. Shao ${ }^{6}$ \\ ${ }^{1}$ LAL, Université Paris-Sud, CNRS/IN2P3, 91406 Orsay, France \\ ${ }^{2}$ IPNO, Université Paris-Sud, CNRS/IN2P3, 91406 Orsay, France \\ ${ }^{3}$ FNSPE, Czech Technical University, Prague, Czech Republic \\ ${ }^{4}$ Laboratoire Leprince Ringuet, École Polytechnique, CNRS/IN2P3, 91128 Palaiseau, France \\ ${ }^{5}$ Faculty of Physics, Warsaw University of Technology, Ulica Koszykowa 75, 00-662 Warsaw, Poland \\ ${ }^{6}$ PH Department, Theory Unit, CERN, CH-1211, Geneva 23, Switzerland
}

Correspondence should be addressed to B. Trzeciak; trzecbar@fffi.cvut.cz

Received 17 April 2015; Accepted 16 June 2015

Academic Editor: Michal Kreps

Copyright (C) 2015 L. Massacrier et al. This is an open access article distributed under the Creative Commons Attribution License, which permits unrestricted use, distribution, and reproduction in any medium, provided the original work is properly cited. The publication of this article was funded by SCOAP $^{3}$.

Being used in the fixed-target mode, the multi-TeV LHC proton and lead beams allow for studies of heavy-flavour hadroproduction with unprecedented precision at backward rapidities, far negative Feynman- $x$, using conventional detection techniques. At the nominal LHC energies, quarkonia can be studied in detail in $p+p, p+d$, and $p+A$ collisions at $\sqrt{s_{\mathrm{NN}}} \simeq 115 \mathrm{GeV}$ and in $\mathrm{Pb}+p$ and $\mathrm{Pb}+A$ collisions at $\sqrt{s_{\mathrm{NN}}} \simeq 72 \mathrm{GeV}$ with luminosities roughly equivalent to that of the collider mode that is up to $20 \mathrm{fb}^{-1} \mathrm{yr}^{-1}$ in $p+p$ and $p+d$ collisions, up to $0.6 \mathrm{fb}^{-1} \mathrm{yr}^{-1}$ in $p+A$ collisions, and up to $10 \mathrm{nb}^{-1} \mathrm{yr}^{-1}$ in $\mathrm{Pb}+A$ collisions. In this paper, we assess the feasibility of such studies by performing fast simulations using the performance of a LHCb-like detector.

\section{Introduction}

Since its start-up, the large hadron collider (LHC), the most energetic hadron collider ever built so far, has already made the demonstration of its outstanding capabilities. These can greatly be complemented by the addition of a fixed-target physics program. Its multi-TeV beams indeed allow one to study $p+p, p+d$, and $p+A$ collisions at a center-ofmass (c.m.s.) energy $\sqrt{s_{\mathrm{NN}}} \simeq 115 \mathrm{GeV}$ as well as $\mathrm{Pb}+p$ and $\mathrm{Pb}+A$ collisions at $\sqrt{s_{\mathrm{NN}}} \simeq 72 \mathrm{GeV}$, with the high precision typical of the fixed-target mode. In this context, the proposal of a fixed-target experiment at the LHC [1], referred to as AFTER@LHC, has been promoted [1] in order to complement the existing collider experiments such as the Relativistic Heavy Ion Collider (RHIC) or the future Electron-Ion Collider (EIC) project in a similar energy range. The idea underlying the AFTER@LHC proposal is a multipurpose detector allowing for the study of a multitude of probes.

Various technological ways to perform fixed-target experiment at the LHC exist. On the one hand, the beam can be extracted by means of a bent crystal. This technology $[2,3]$ is currently developed as a smart beam-collimation solution and is studied by the UA9/LUA9 collaboration, respectively, at SPS and LHC. A bent crystal installed in the halo of the LHC beam would deflect the particles of the halo onto a target, with a flux of $5 \times 10^{8}$ proton/s without any impact on the LHC performances [3-5].

On the other hand, the LHC beam can go through an internal-gas-target system in an existing (or new) LHC experiment. Such a system is already tested at low gas pressure by the LHCb collaboration to monitor the luminosity of the beam [6-8]. Data were taken at a center-of-mass energy of 
TABLE 1: Expected luminosities obtained for a 7 (2.76) TeV proton beam $(\mathrm{Pb})$ extracted by means of a bent crystal or obtained with an internal-gas-target system.

\begin{tabular}{lccccc}
\hline Beam & Target & $\begin{array}{c}\text { Thickness } \\
(\mathrm{cm})\end{array}$ & $\begin{array}{c}\rho \\
\left(\mathrm{g} \cdot \mathrm{cm}^{-3}\right)\end{array}$ & $\begin{array}{c}\mathscr{L} \\
\left(\mu \mathrm{b}^{-1} \cdot \mathrm{s}^{-1}\right)\end{array}$ & $\begin{array}{c}\text { L } \\
\left(\mathrm{pb}^{-1} \cdot \mathrm{y}^{-1}\right)\end{array}$ \\
\hline $\mathrm{p}$ & Liquid $\mathrm{H}$ & 100 & 0.068 & 2000 & 20000 \\
$\mathrm{p}$ & Liquid $\mathrm{D}$ & 100 & 0.16 & 2400 & 24000 \\
$\mathrm{p}$ & $\mathrm{Pb}$ & 1 & 11.35 & 16 & 160 \\
\hline $\mathrm{Pb}$ & Liquid $\mathrm{H}$ & 100 & 0.068 & 0.8 & 0.8 \\
$\mathrm{~Pb}$ & Liquid $\mathrm{D}$ & 100 & 0.16 & 1 & 1 \\
$\mathrm{~Pb}$ & $\mathrm{~Pb}$ & 1 & 11.35 & 0.007 & 0.007 \\
\hline $\mathrm{Beam}$ & Target & Usable gas zone & Pressure & $\mathscr{L}$ & $\int \mathscr{L}$ \\
\hline $\mathrm{p}$ & Perfect gas & 100 & $10^{-9}$ & 10 & 100 \\
\hline $\mathrm{Pb}$ & Perfect gas & 100 & $10^{-9}$ & 0.001 & 0.001 \\
\hline
\end{tabular}

$\sqrt{s_{\mathrm{NN}}}=87(54) \mathrm{GeV}$ with $p+\mathrm{Ne}(\mathrm{Pb}+\mathrm{Ne})$ collisions during pilot runs in 2012 and 2013. Although this system, called SMOG, was tested during only few hours in a row, no decrease of the LHC performances was observed.

In the bent crystal case, the luminosity achievable with AFTER@LHC would surpass that of RHIC by 3 orders of magnitudes [1]. We have reported in Table 1 the instantaneous and yearly integrated luminosities expected with the proton and $\mathrm{Pb}$ beams on various target species of various thicknesses, for the bent crystal as well as internal-gas-target options. Integrated luminosities as large as $20 \mathrm{fb}^{-1}$ can be delivered during a one-year run of $p+\mathrm{H}$ collisions with a bent crystal. Besides, it is worth mentioning that both technologies allow one to polarise the target, which is an important requirement to lead an extensive spin physics programme $[1,11]$.

Overall, thanks to the large luminosity expected, AFTER@LHC would become a quarkonium [12], prompt photon, and heavy-flavour observatory $[1,13]$ in $p+p$ and $p+A$ collisions where, by instrumenting the target-rapidity region, gluon and heavy-quark distributions of the proton, the neutron and the nuclei can be accessed at large $x$ and even at $x$ larger than unity in the nuclear case [14]. In addition, the fixed-target mode allows for single-target-spin-asymmetry measurements over the full backward rapidity domain up to $x_{F} \simeq-1[15,16]$. Also, the versatility in the target choices offer a unique opportunity to study the nuclear matter versus the hot and dense matter formed in heavy ion collisions which can be studied during the one-month lead run. In the latter case, modern detection technology (such as high granularity calorimeter) should allow for extensive studies of quarkonium excited states, from $\psi(2 S)$ to $\chi_{c}$ and $\chi_{b}$ resonances thanks to the boost of the fixed-target mode [17].

In this paper, we report on a feasibility study of quarkonium production at a fixed-target experiment using LHC beams. In Section 2, we outline the simulation framework which was used. In Section 3, we describe how a fast simulation of a detector response has been implemented, following a LHCb-like detector setup. In Section 4, we present the charmonium and bottomonium family studies performed with the $p+\mathrm{H}$ simulations at $\sqrt{s}=115 \mathrm{GeV}$. In Section 5, we present multiplicity studies in $p+A$ and $A+p$ collisions as well as the expected nuclear modification factors for $J / \psi$ and $\Upsilon$ in $p+\mathrm{Pb}$ collisions at $\sqrt{s_{\mathrm{NN}}}=115 \mathrm{GeV}$. Finally in Section 6 some prospects for $\mathrm{Pb}+A$ measurement at $\sqrt{s_{\mathrm{NN}}}=72 \mathrm{GeV}$ are given. Section 7 gathers our conclusions.

\section{Simulation Inputs}

In order to get the most realistic minimum bias simulations at AFTER@LHC energy for quarkonium studies in the dimuon decay channels, we have simulated the quarkonium signal and all the background sources separately to have under control the transverse momentum and rapidity input distributions as well as the normalisation of the different sources.

The simulation has been performed for $p+p$ collisions at $\sqrt{s}=115 \mathrm{GeV}$. On the one hand, the quarkonium signal and the correlated background (Drell-Yan, $c \bar{c}, b \bar{b}$ ) were simulated with HELAC-ONIA [18] which produces outputs following the format of Les Houches Event Files [19]. The outputs were then processed with Pythia (Pythia 8.185 [20]) to perform the hadronisation, the initial/final-state radiations, and the decay of the resonances. On the other hand, the uncorrelated background was obtained from minimum bias $p+p$ collisions generated with PyтHIA.

The relative normalisation of the signal and background sources was performed according to the production cross section of the process (taking into account initial phase space cuts, if any). Values of the cross section and the number of simulated events $N_{\text {sim }}$, not to be confused with the expected events for a specific luminosity, are reported in Table 2 . The cross section values are integrated over rapidity and $p_{T}$.

\subsection{Signal and Correlated Background}

2.1.1. Quarkonium Signal. $J / \psi, \psi(2 S), \Upsilon(1 S), \Upsilon(2 S)$, and $\Upsilon(3 S)$ were simulated in a data-driven way. The amplitude of $g g \rightarrow \mathbb{Q}+X$ (where $\mathbb{Q}$ is the quarkonium) is expressed in an empirical functional form [21]:

$$
\begin{aligned}
& \overline{\left|\mathscr{A}_{g g \rightarrow Q+X}\right|^{2}} \\
& = \begin{cases}K \exp \left(-\kappa \frac{p_{T}^{2}}{M_{\mathscr{Q}}^{2}}\right) & \text { when } p_{T} \leq\left\langle p_{T}\right\rangle \\
K \exp \left(-\kappa \frac{\left\langle p_{T}\right\rangle^{2}}{M_{\mathscr{Q}}^{2}}\right)\left(1+\frac{\kappa}{n} \frac{p_{T}^{2}-\left\langle p_{T}\right\rangle^{2}}{M_{\mathscr{Q}}^{2}}\right)^{-n} & \text { when } \left.p_{T}\right\rangle\left\langle p_{T}\right\rangle,\end{cases}
\end{aligned}
$$

where $K=\lambda^{2} \kappa \widehat{s} / M_{\mathscr{Q}}^{2}$ with $\widehat{s}$ the partonic center-of-mass energy and $M_{\mathscr{Q}}$ the mass of the quarkonium $Q$ taken from the PDG table [22].

The parameters $\kappa, \lambda, n$, and $\left\langle p_{T}\right\rangle$ were determined by fitting the differential cross section $d^{2} \sigma / d p_{T} d y$ to the experimental data. The dedicated codes used to perform the fit and to generate unweighted events for quarkonium production have been implemented in HELAC-ONIA [18] and we used MSTW2008NLO PDF set [23] provided in LHAPDF5 [24] and the factorisation scale $\mu_{F}=\sqrt{M_{Q}^{2}+p_{T}^{2}}$. In order to constrain the nontrivial energy dependence of quarkonium production, we used the differential measurements of charmonium production performed by the 


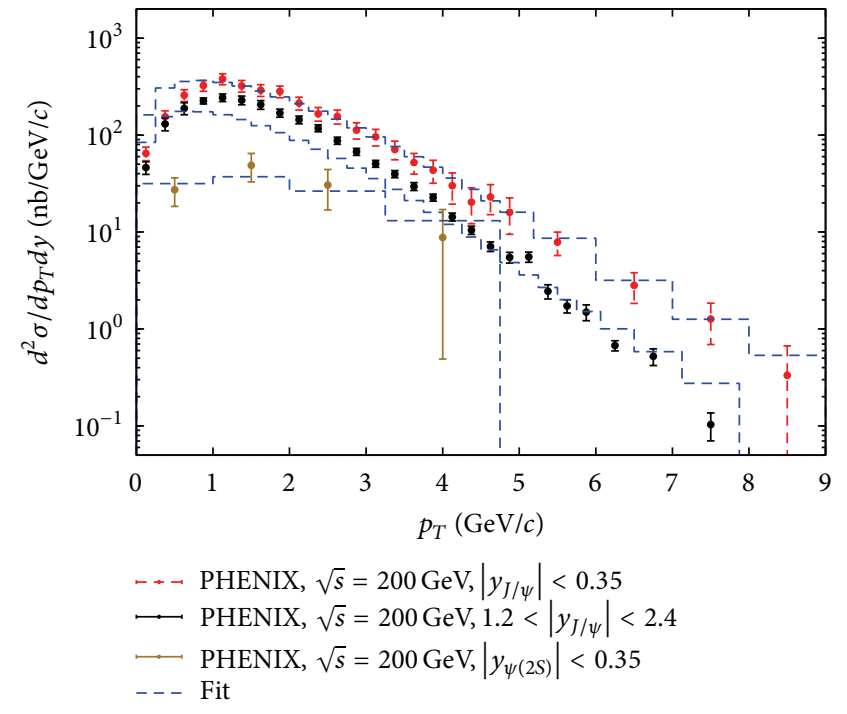

(a)

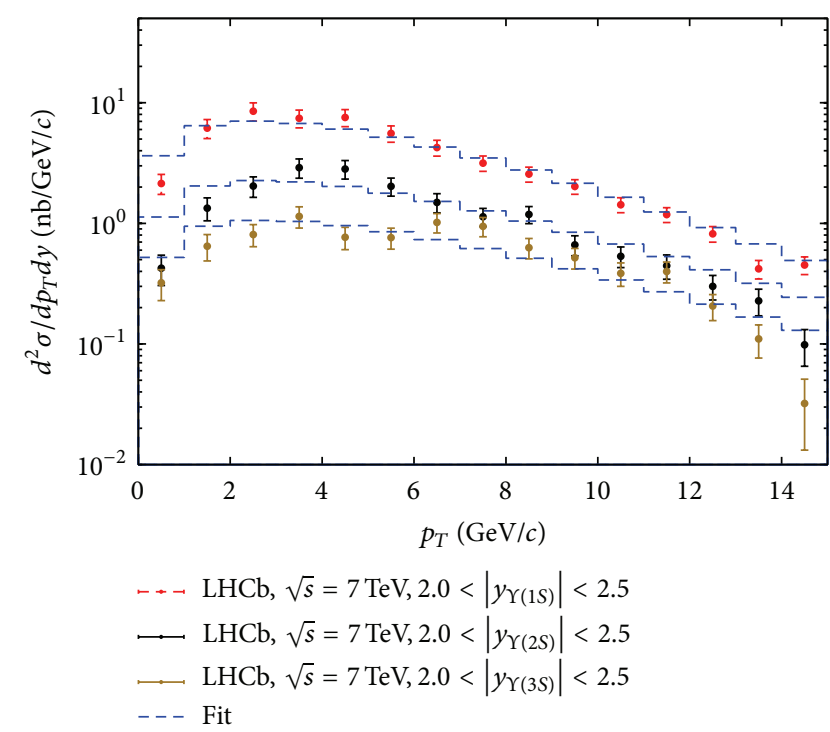

(b)

FIgURE 1: Some illustrative comparisons between fits and the PHENIX data [9] for charmonium production (a) and LHCb data [10] for bottomonium production (b).

TABLE 2: Total cross section for different processes in $p+p$ collisions at $\sqrt{s}=115 \mathrm{GeV}$ and number of simulated events $N_{\text {sim }}$.

\begin{tabular}{lcc}
\hline & $\sigma_{\text {tot }}(\mathrm{mb})$ & $N_{\text {sim }}$ \\
\hline$J / \psi$ & $1.30 \times 10^{-3}$ & $1.47 \times 10^{6}$ \\
$\psi(2 S)$ & $1.61 \times 10^{-4}$ & $1.12 \times 10^{6}$ \\
$Y(1 S)$ & $4.30 \times 10^{-7}$ & $1.46 \times 10^{6}$ \\
$Y(2 S)$ & $1.22 \times 10^{-7}$ & $1.49 \times 10^{6}$ \\
$Y(3 S)$ & $5.28 \times 10^{-8}$ & $1.48 \times 10^{6}$ \\
Drell-Yan $\left(M>2.5 \mathrm{GeV} / c^{2}\right)$ & $2.52 \times 10^{-6}$ & $4.3 \times 10^{5}$ \\
Drell-Yan $\left(M>7 \mathrm{GeV} / c^{2}\right)$ & $1.49 \times 10^{-7}$ & $2.0 \times 10^{6}$ \\
$c \bar{c}$ & $2.29 \times 10^{-1}$ & $81.5 \times 10^{6}$ \\
$b \bar{b}$ & $4.86 \times 10^{-4}(g g \rightarrow b \bar{b})$ & $32.3 \times 10^{6}(g g \rightarrow b \bar{b})$ \\
Minimum bias & $1.49 \times 10^{-4}(q \bar{q} \rightarrow b \bar{b})$ & $85.7 \times 10^{6}(q \bar{q} \rightarrow b \bar{b})$ \\
\hline
\end{tabular}

PHENIX collaboration at RHIC, in $p+p$ collisions at $\sqrt{s}=$ $200 \mathrm{GeV}$ [9] to predict the corresponding yields at $\sqrt{s}=$ $115 \mathrm{GeV}$. Given the lack of such measurements for $\Upsilon$ at RHIC, we performed a combined fit to CDF [25], ATLAS [26], CMS [27], and $\mathrm{LHCb}[10,28]$ data on $\Upsilon$ production. The values of the fitted parameters are listed in Table 3. For illustration, the comparison between fits and the selected experimental data is shown in Figure 1.

In order to increase the statistics of the simulated data sample, the decay of the quarkonium in PүтніA is forced into the dimuon decay channel. The simulated yields are then weighted by the cross section for this process multiplied by the Branching Ratio (BR).

2.1.2. Open Charm. Open charm production was simulated with the process $g g \rightarrow c \bar{c}$ in HELAC-ONIA. In order to avoid the huge theoretical uncertainties in the state-of-theart perturbative calculations, open charm yields at $\sqrt{s}=$
TABLE 3: Fit parameters obtained after a combined fit of $d^{2} \sigma / d p_{T} d y$ to the PHENIX data [9] for charmonium production and to CDF [25], ATLAS [26], CMS [27], and LHCb [10, 28] data for bottomonium production. We have fixed $n=2$ and $\left\langle p_{T}\right\rangle=4.5$ (13.5) $\mathrm{GeV} / c$ for charmonium (bottomonium) production. The number of fitted data points is also reported.

\begin{tabular}{ccccc}
\hline & $\kappa$ & $\lambda$ & Number of data points & $\chi^{2}$ \\
\hline$J / \psi$ & 0.674 & 0.380 & 51 & 422 \\
$\psi(2 S)$ & 0.154 & 0.351 & 4 & 1.12 \\
$\Upsilon(1 S)$ & 0.707 & 0.0837 & 288 & 1883 \\
$\Upsilon(2 S)$ & 0.604 & 0.0563 & 205 & 856 \\
$\Upsilon(3 S)$ & 0.591 & 0.0411 & 197 & 886 \\
\hline
\end{tabular}

$115 \mathrm{GeV}$ are also computed in a data-driven way following the method described in the previous section. Similarly, the matrix element of $g g \rightarrow c \bar{c}$ is determined using (1). 
The parameters are obtained from a fit to the $p_{T}$-differential $c \bar{c}$ cross section measured by the STAR experiment [29] in $p+p$ collisions at $\sqrt{s}=200 \mathrm{GeV}$ (see Figure 3 ). We obtained $\kappa=0.437, \lambda=3.04$, and $\left\langle p_{T}\right\rangle=2.86 \mathrm{GeV} / c$ when $n=2$ by using CTEQ6L1 [30] and by fixing the $c$ quark mass to $m_{c}=$ $1.5 \mathrm{GeV} / c^{2}$ and the factorisation scale to $\mu_{F}=\sqrt{m_{c}^{2}+p_{T}^{2}}$. The $\chi^{2}$ of the fit is equal to 4.39 with 10 experimental data points. The tuned result is shown in Figure 2. The evolution of the cross section with the energy down to $\sqrt{s}=115 \mathrm{GeV}$ is then given by HELAC-ONIA.

After embedding the Les Houches Event File into PyтнiA, muons from the underlying Pyтнia event can be produced on top of muons from the initial $c \bar{c}$ pair. The combination of those additional muons with a muon from the initial $c \bar{c}$ pair is not included in our definition of open charm correlated background. We have however checked that this contribution is negligible. In order to increase the statistics, $D^{0}, \overline{D^{0}}, D^{+/-}$, and $D_{s}^{+/-}$were forced to decay into muons and only those decay muons were considered as correlated background. $\mu^{+} \mu^{-}$pairs coming from all possible combinations, $D^{0} \overline{D^{0}}, D^{+} D^{-}, D_{s}^{+} D_{s}^{-}, D^{0} D^{+/-}, D^{0} D_{s}^{+/-}$, and $D^{+/-} D_{s}^{-/+}$, are considered. The simulated events are weighted by the production cross section times the pair Branching Ratio times the fraction of $c$ quark fragmenting to $D^{0}, \overline{D^{0}}, D^{+/-}$, or $D_{s}^{+/-}$. This fraction is obtained from PyтHIA and found to be $95 \%$.

2.1.3. Open Beauty. The theoretical uncertainty on open beauty production is relatively smaller than the one on open charm production. We therefore calculated open beauty production yields with a Leading Order (LO) matrix element which was normalised to the Next-To-Leading-Order (NLO) $K$ factor. The NLO cross section with the same setup was calculated by MADGRAPH5_AMC@NLO [31]. We used CTEQ6L1 (CTEQ6M) for the LO (NLO) calculation. The $K$ factor is found to be 1.83 . The renormalisation and factorisation scale is $\mu_{R}=\mu_{F}=\sqrt{m_{b}^{2}+p_{T}^{2}}$ with the mass of the $b$ quark taken as $m_{b}=4.5 \mathrm{GeV} / c^{2}$. We have adopted a similar definition for the open beauty correlated background as the one of open charm (see the previous section).

2.1.4. Drell-Yan. Drell-Yan (DY) correlated background was simulated with the process $q \bar{q} \rightarrow \gamma^{*} / Z \rightarrow \mu^{+} \mu^{-}$at LO where $q \bar{q}$ is a pair of the same flavour light quarks. The LO calculation was done with the CTEQ6L1 pdf set and the renormalisation and factorisation scale was set to $\mu_{R}=$ $\mu_{F}=Q / 2$. In order to have enough statistics in the $J / \psi$ and $\psi(2 S)$ mass window, a phase space cut requesting that the invariant mass of the dimuons $(M)$ is greater than $2.5 \mathrm{GeV} / c^{2}$ was applied. For the simulation of the DY background under the $\Upsilon$ family peaks, a phase space cut $M>7 \mathrm{GeV} / c^{2}$ was applied. The DY cross section obtained with HELAC-ONIA at $\sqrt{s}=38.8 \mathrm{GeV}$ is compared to the existing E866 data at the same energy [32]. A $K$ factor 1.2 is needed to match the data and therefore it was also applied at $\sqrt{s}=115 \mathrm{GeV}$. Such a $K$ factor is known to approximately account for the higherorder QCD corrections.

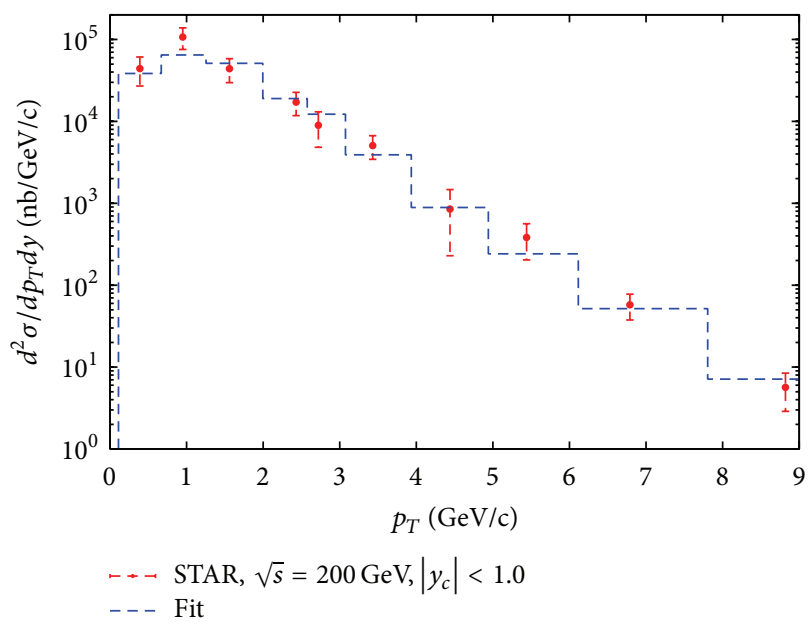

FIgURE 2: A comparison between fit and the STAR data [29] in $p+p$ collisions at $\sqrt{s}=200 \mathrm{GeV}$, for $c \bar{c}$ production.

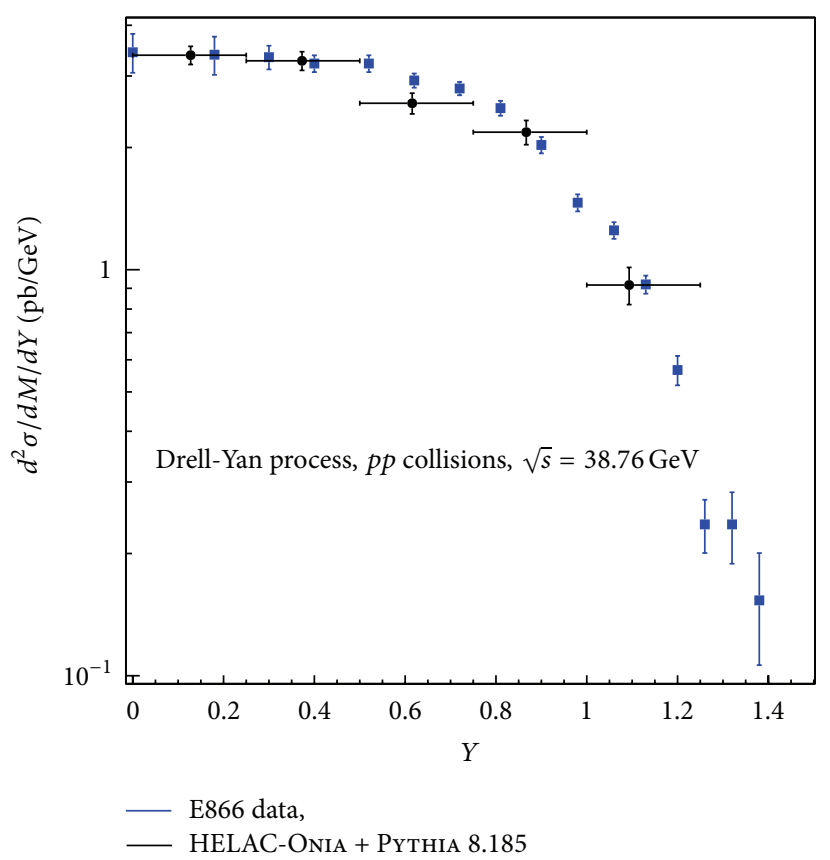

FIGURE 3: Drell-Yan cross section as a function of the rapidity in the center-of-mass frame obtained with HELAC-ONIA + PYTHIA at $\sqrt{s}=38.76 \mathrm{GeV}$ and rescaled by a factor 1.2, together with E866 data extracted from [33]. The invariant mass range considered is $7.2<$ $M<8.7 \mathrm{GeV} / c^{2}$.

2.2. Uncorrelated Background. The uncorrelated background was obtained from a minimum bias Pүтнia $p+p$ simulation at $\sqrt{s}=115 \mathrm{GeV}$ using the process SoftQCD:nonDiffractive with the MRSTMCal.LHgrid LHAPDF (6.1.4) set [34]. By comparing our simulation of open charm with a low statistic pure minimum bias PyтнiA simulation, we have checked that the contribution of dimuons originating from a muon from charm/beauty and a muon from $\pi / K$ is negligible. 


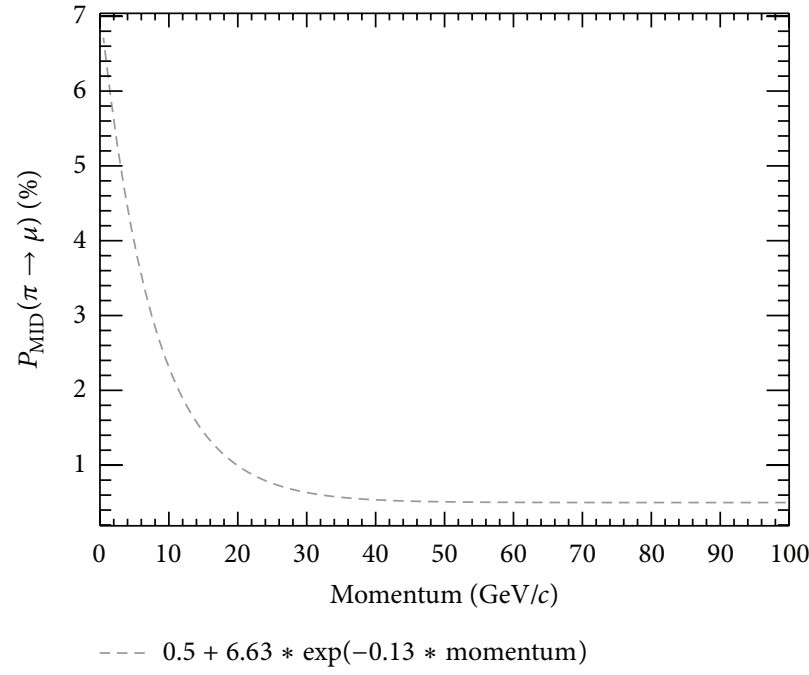

(a)

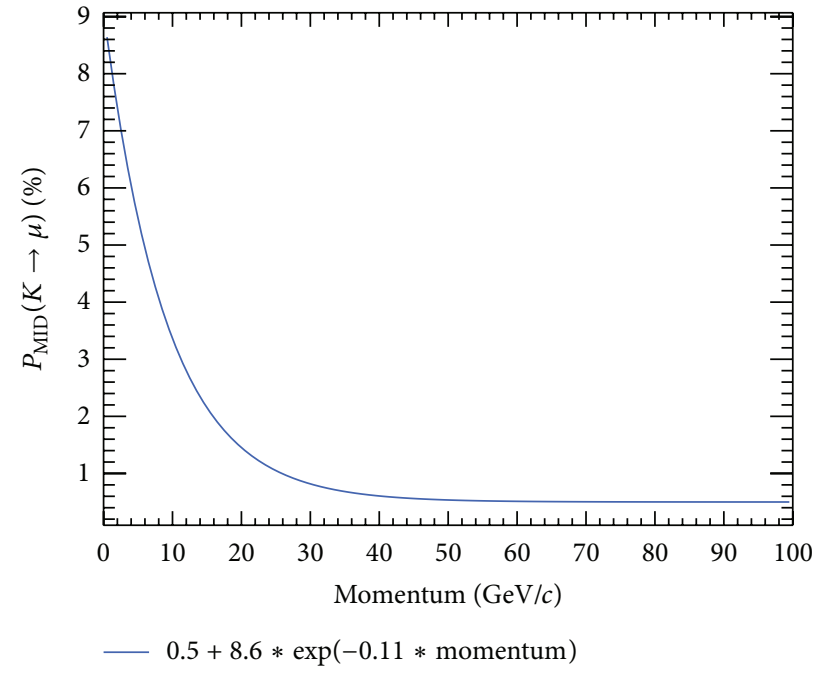

(b)

Figure 4: Misidentification probability of $\pi(\mathrm{a})$ and $K(\mathrm{~b})$ as muon candidates as a function of momentum, $P_{\mathrm{MID}}(\pi \rightarrow \mu)$ and $P_{\mathrm{MID}}(K \rightarrow \mu)$, respectively.

The dominant source of uncorrelated opposite-sign muon pairs is the simultaneous semimuonic decay of uncorrelated $\pi$ and/or $K$. In order to avoid possible double counting of signal and correlated background processes, the following hard processes have been switched off from the minimum bias simulations: HardQCD : hardccbar, HardQCD: hardbbbar, WeakSingleBoson:ffbar2gmZ (in order to avoid Drell-Yan pair production.), Charmonium:all and Bottomonium:all,.

\section{Fast Simulation of the Response of a LHCb-Like Detector}

The HELAC-Onia and Pythia generators provide the opposite-sign muon pairs from quarkonium decays, correlated and uncorrelated backgrounds sources, as defined in the previous section. In order to account for the detector resolution and the particle identification capabilities of a given detector and to investigate the feasibility of the quarkonium studies in $p+p$ collisions at $\sqrt{s} \simeq 115 \mathrm{GeV}$, the detector response needs to be simulated. For this purpose, we have chosen a detector setup similar to the LHCb detector [35]. A forward detector is very well suited as a fixed-target experiment setup as well, with a good tracking and particle identification capabilities.

According to LHCb analysis cuts, muons in our simulations are required to have their transverse momentum satisfying $p_{T}>0.7 \mathrm{GeV} / c$ [36] and their pseudorapidity in the laboratory frame satisfying $2<\eta<5$. The $\eta$ cut range corresponds to the $\mathrm{LHCb}$ detector coverage. Since the momentum resolution reported by LHCb is $\delta p / p \sim 0.4$ $(0.6) \%$ for a momentum of $3(100) \mathrm{GeV} / c$ [37], we consider a momentum resolution of $\delta p / p=0.5 \%$. The single $\mu$ identification efficiency is taken to be $\epsilon_{P}=98 \%$, which is an average efficiency obtained by LHCb for muons coming from $J / \psi$ decays, for $p>3 \mathrm{GeV} / c$ and $p_{T}>0.8 \mathrm{GeV} / c$ [37].
These cuts and the abovementioned detector response on the muons are applied to simulate the quarkonium states and all the background sources.

In the case of uncorrelated background, as discussed in Section 2, most of the $\mu$ originate from $\pi^{+/-}$or $\mathrm{K}^{+/-}$ decays. If a $\pi$ or $K$ decays to a $\mu$ before $12 \mathrm{~m}$ along the $z$ axis, the $\mu$ is rejected by the tracking system and it is not considered in the simulation. $12 \mathrm{~m}$ corresponds to the distance where the calorimeters, followed by the muon stations, are placed in the LHCb detector setup. If the $\mu$ is produced beyond $12 \mathrm{~m}$ or if a $\pi / K$ is misidentified with $\mu$ in the muon stations, a $\pi / K$ misidentification probability is applied. The misidentification probabilities depend on the total particle momentum and were reported by the LHCb collaboration in [38]. These probabilities are parameterised with the following functions: $P_{\mathrm{MID}}(\pi \rightarrow \mu)(p)=$ $(0.5+6.63 \exp (-0.13 p)) \%$ and $P_{\mathrm{MID}}(K \rightarrow \mu)(p)=$ $(0.5+8.6 \exp (-0.11 p)) \%$, and they are shown in Figures $4(\mathrm{a})$ and 4 (b), for $\pi$ and $K$, respectively. Based on the single $\mu$ identification efficiency $\epsilon_{\mu^{+/-}}$, the dimuon, $\mu^{+} \mu^{-}$, efficiency is calculated as a product of the single efficiencies: $\epsilon_{\mu^{+} \mu^{-}}=\epsilon_{\mu^{+}} \times \epsilon_{\mu^{-}}$. For muons coming from $\pi^{+}$or $\mathrm{K}^{+/-}$ decays, misidentification probabilities are used: $\epsilon_{\mu^{+/}}=$ $P_{\mathrm{MID}}(\pi \rightarrow \mu)(p)$ or $\epsilon_{\mu^{+/-}}=P_{\mathrm{MID}}(K \rightarrow \mu)(p)$, respectively, for $\pi$ and $K$, and for prompt muons $\epsilon_{\mu^{+/-}}=\epsilon_{P}=0.98$.

The pair efficiency is extracted in each kinematic phase space point and is shown as a function of the dimuon invariant mass, transverse momentum, and rapidity in Figure 5. This efficiency is used to correct dimuon spectra obtained with the uncorrelated background PутнIA simulations.

\section{Quarkonium Production Studies in $p+\mathbf{H}$ Collisions at $\sqrt{s}=115 \mathbf{~ G e V}$}

In this section, we show results on the quarkonium production studies in the dimuon decay channels, with 


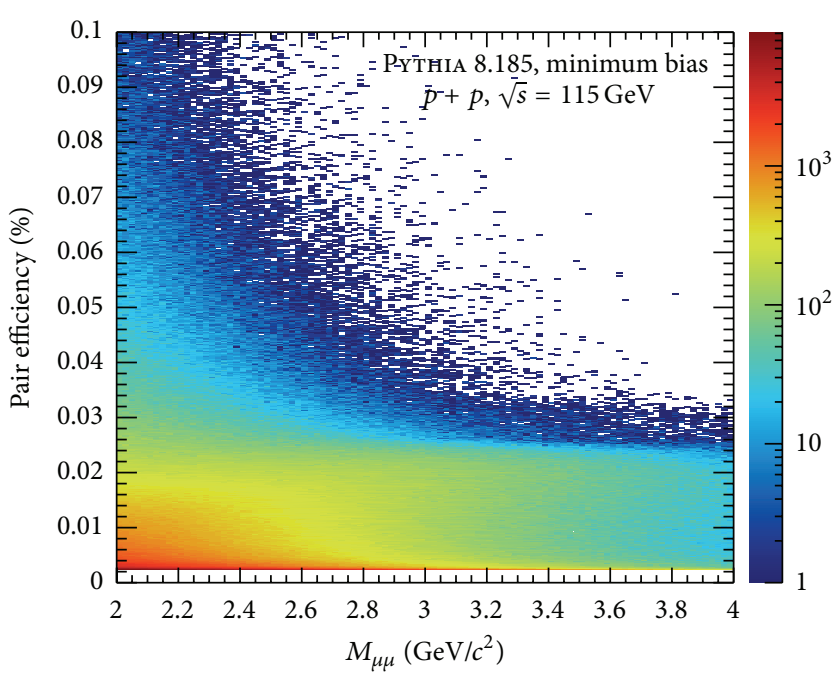

(a)

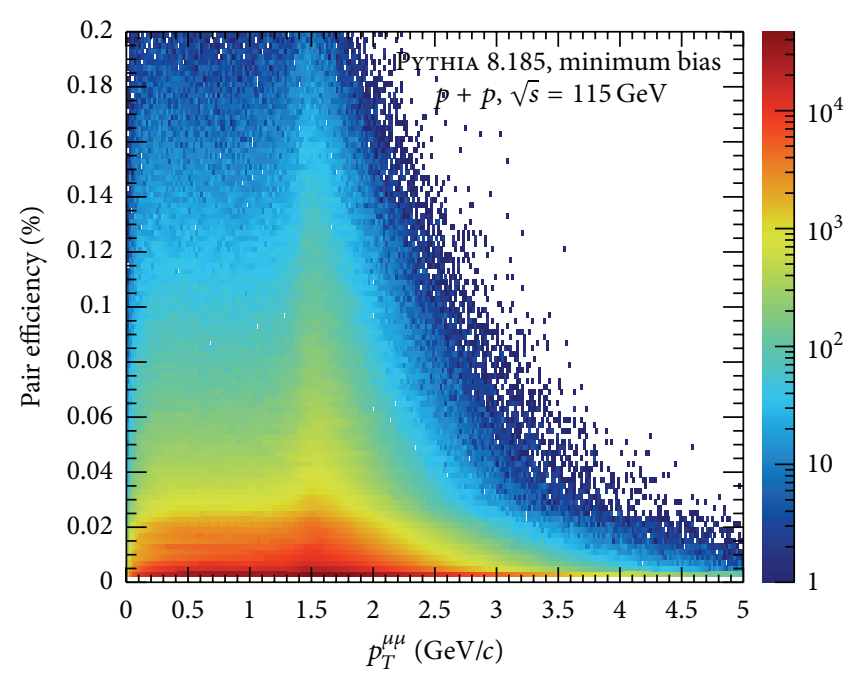

(b)

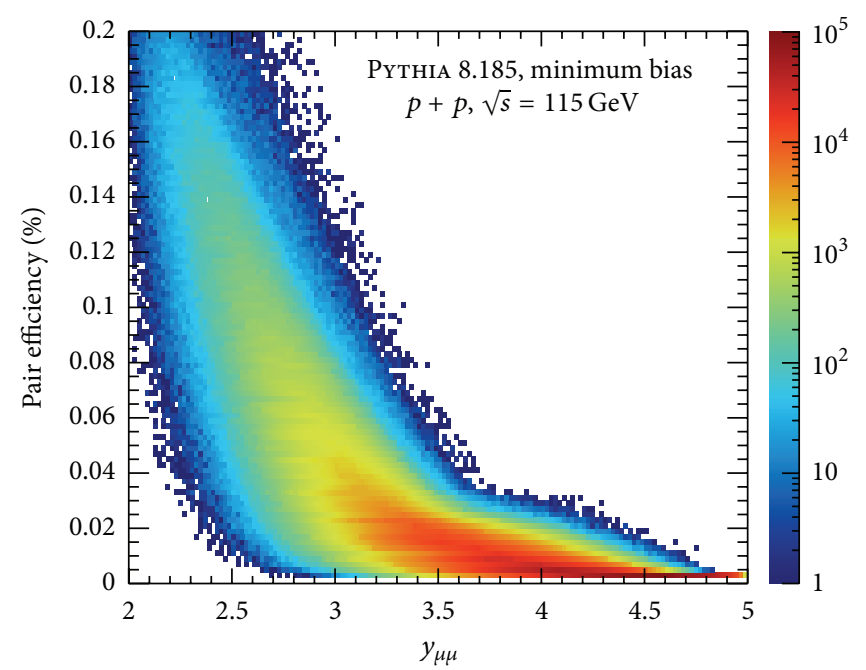

(c)

Figure 5: Muon pair, $\mu^{+} \mu^{-}$, identification efficiency as a function of the pair invariant mass (a), transverse momentum (b), and rapidity (c) for uncorrelated muon background. The efficiency takes into account the identification efficiency of the prompt muons and the $\pi$ and $K$ misidentification probability, $P_{\mathrm{MID}}(\pi \rightarrow \mu)$ and $P_{\mathrm{MID}}(K \rightarrow \mu)$.

the dominant background sources. Simulations have been performed for a $7 \mathrm{TeV}$ proton beam on a hydrogen target $(p+p)$, which gives $\sqrt{s}=115 \mathrm{GeV}$. We consider an integrated luminosity of $10 \mathrm{fb}^{-1}$ which is expected to be obtained after half of a LHC year with the crystal mode, as described in Section 1 and Table 1.

4.1. Background Studies. These simulations allow us to quantify the background sources in the quarkonium studies in the dimuon decay channel, which could potentially make the quarkonium signal extraction more difficult or even prevent from obtaining a clear signal. In particular, this may be critical for the excited states. We present here simulations of invariant mass of opposite-sign muon pairs, $\mu^{+} \mu^{-}$, from the quarkonia and from the dominant background sources, in two mass ranges; see Figure 6 . The first range corresponds to the $J / \psi$ and $\psi(2 S)$ invariant mass windows and the second one to the mass range of the $\Upsilon(1 S), \Upsilon(2 S)$, and $\Upsilon(3 S)$. The invariant mass distributions are integrated over the whole transverse momentum and rapidity ranges. The plots show the simulated quarkonium signals and the background, separately from the different sources, and the black solid line is a sum of all contributions. The background sources correspond to an integrated luminosity of $10 \mathrm{fb}^{-1}$.

In the $J / \psi$ and $\psi(2 S)$ invariant mass window, the dominant background source is from uncorrelated $\mu^{+} \mu^{-}$pairs, mostly from $\pi^{+/-}$and $K^{+/-}$decays. The contributions from Drell-Yan and $b \bar{b}$ continuum are very small. In the case of $\Upsilon(n S)$ states, the Drell-Yan contribution is the dominant one. Under the $\Upsilon(n S)$ peak, the contribution from the $c \bar{c}$ continuum is negligible, and it is not considered here. 


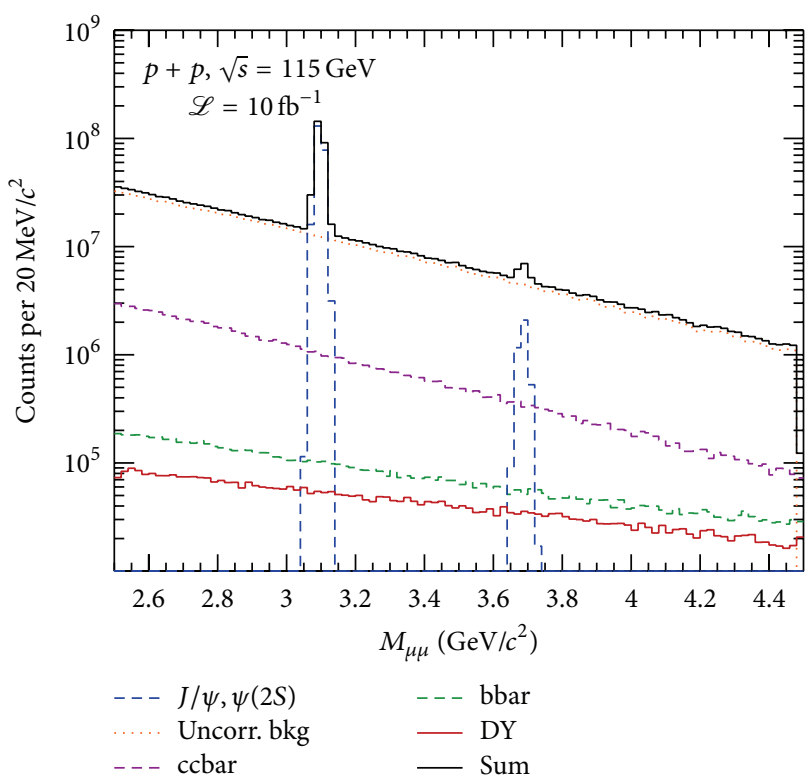

(a)

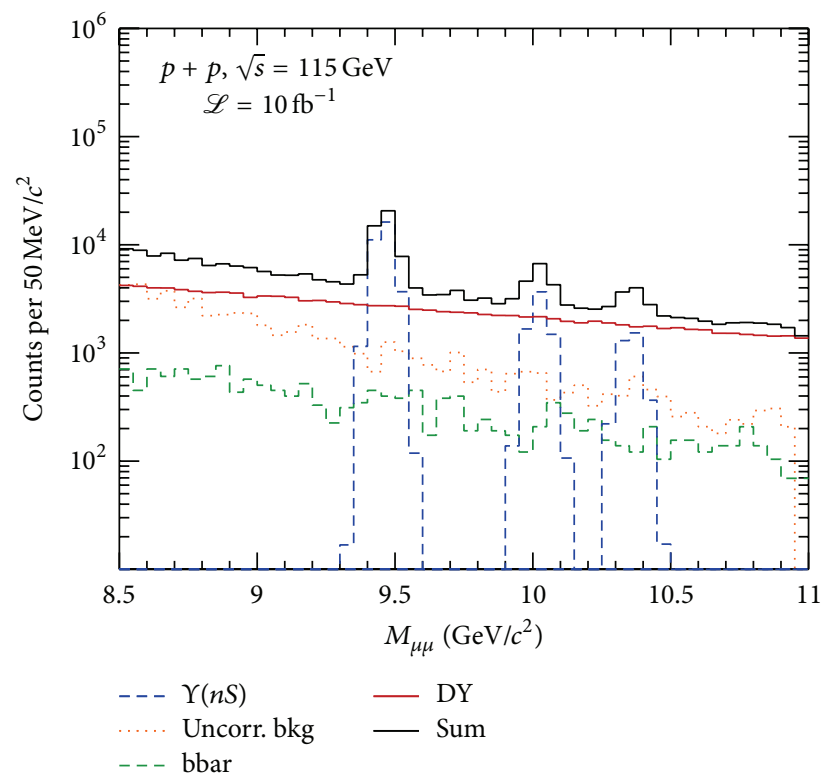

(b)

FIGURE 6: Dimuon invariant mass distributions for $J / \psi$ and $\psi(2 S)$ (a) and $\Upsilon(n S)$ (b) with different background sources.

The significance $(\operatorname{sig}=S / \sqrt{(S+B)}$, where $S$ is the number of signal counts and $B$ is the number of background counts, in the invariant mass range $M_{\mathrm{Q}} \pm 3 \sigma_{\mathrm{Q}}$ ) and the signal to background ratio $(S / B)$ of each quarkonium state are given in the following:

(i) $\operatorname{sig}_{J / \psi}=134.610^{2} \sigma, S / B_{J / \psi}=4.21$,

(ii) $\operatorname{sig}_{\psi(2 S)}=735.2 \sigma, S / B_{\psi(2 S)}=0.16$,

(iii) $\operatorname{sig}_{\Upsilon(1 S)}=140.73 \sigma, S / B_{\Upsilon(1 S)}=1.75$,

(iv) $\operatorname{sig}_{\Upsilon(2 S)}=45.29 \sigma, S / B_{\Upsilon(2 S)}=0.48$,

(v) $\operatorname{sig}_{\Upsilon(3 S)}=25.75 \sigma, S / B_{\Upsilon(3 S)}=0.28$,

for $J / \psi, \psi(2 S), \Upsilon(1 S), \Upsilon(2 S)$, and $\Upsilon(3 S)$, respectively.

Transverse momentum and rapidity distributions for the quarkonium signals and for each background source were also studied. As an example, the $p_{T}$ and $y$ distributions in the $J / \psi$ mass range, $3.063<M_{\mu^{+} \mu^{-}}<3.129 \mathrm{GeV} / c^{2}$ (corresponding to $M_{J / \psi} \pm 3 \sigma_{J / \psi}$ ), are shown in Figure 7 . It is visible that the distributions for the $J / \psi$ and different backgrounds differ. In more backward or forward rapidity regions, the signal to background ratio increases. This can also be seen in Figure 8, where the dimuon invariant mass distributions in $J / \psi$ and $\psi(2 S)$ mass window are shown in three rapidity ranges. In terms of transverse momentum, one can obtain a very clean signal when going to higher $p_{T}$. Above $\sim 4 \mathrm{GeV} / c$, the uncorrelated background starts to vanish. Since $c \bar{c}, b \bar{b}$, and Drell-Yan simulations are LO simulations, the $p_{T}$ spectra of these correlated background sources are not shown here.

4.2. Quarkonium Simulations. We have also studied the $p_{T}$ and rapidity coverage reach of the quarkonium signals. The transverse momentum distributions are shown in
Figure 9 (a), for $J / \psi, \psi(2 S), \Upsilon(1 S), \Upsilon(2 S)$, and $\Upsilon(3 S)$, from the top to the bottom distribution. Similarly, Figure 9(b) shows the rapidity distribution for each quarkonium state. With an integrated luminosity of $10 \mathrm{fb}^{-1}$ the quarkonium studies can be carried out in a wide rapidity and $p_{T}$ range. It should be possible to study $\Upsilon(n S)$ signals up to $p_{T} \simeq 10 \mathrm{GeV} / c$, and $J / \psi$ and $\psi(2 S)$ could be studied even up to $p_{T} \simeq 15 \mathrm{GeV} / c$. All the quarkonium states can be measured down to $p_{T}=0 \mathrm{GeV} / c$.

This study is limited by the rapidity range of $2<y<5$, in the laboratory frame, due to the pseudorapidity cuts on the decay $\mu$. The red $x$-axis on the top of Figure 9 (b) denotes the rapidity in the center-of-mass frame. The rapidity shift for a $7 \mathrm{TeV}$ proton beam on a fixed-target is -4.8 ; that is, $y_{\mathrm{CM}}=$ $0 \rightarrow y_{\mathrm{lab}}=4.8 \mathrm{~J} / \psi$ and $\psi(2 S)$ signals can be studied in the whole mentioned rapidity range, while the lowest rapidity reach for $\Upsilon(n S)$ is $\sim 2.5-3$.

\section{Quarkonium Measurements in $p+A$ Colli- sions at $\sqrt{s}=115 \mathbf{G e V}$ and $\mathbf{P b}+\mathbf{H}$ Collisions at $\sqrt{s}=72 \mathbf{~ G e V}$}

5.1. Multiplicity in Proton-Nucleus Collisions. In protonnucleus collisions, the high track multiplicity may induce a high detector occupancy and lead to a reduction of the detector capabilities. Since LHCb has successfully measured the $J / \psi$ and $\Upsilon$ production in $p+\mathrm{Pb}$ collisions at $\sqrt{s_{\mathrm{NN}}}=$ $5 \mathrm{TeV}[39,40]$, one would expect a good capability of such detector under similar particle multiplicity environment. In the following, the charged particle multiplicity has been generated with the EPOS generator $[41,42]$ in different configurations: $p+\mathrm{Pb}$ collisions at $\sqrt{s_{\mathrm{NN}}}=5 \mathrm{TeV}$ in collider mode (the lead goes in the detector direction), $p+\mathrm{Pb}$ collisions at $\sqrt{s_{\mathrm{NN}}}=115 \mathrm{GeV}$, and $\mathrm{Pb}+\mathrm{H}$ collisions at 


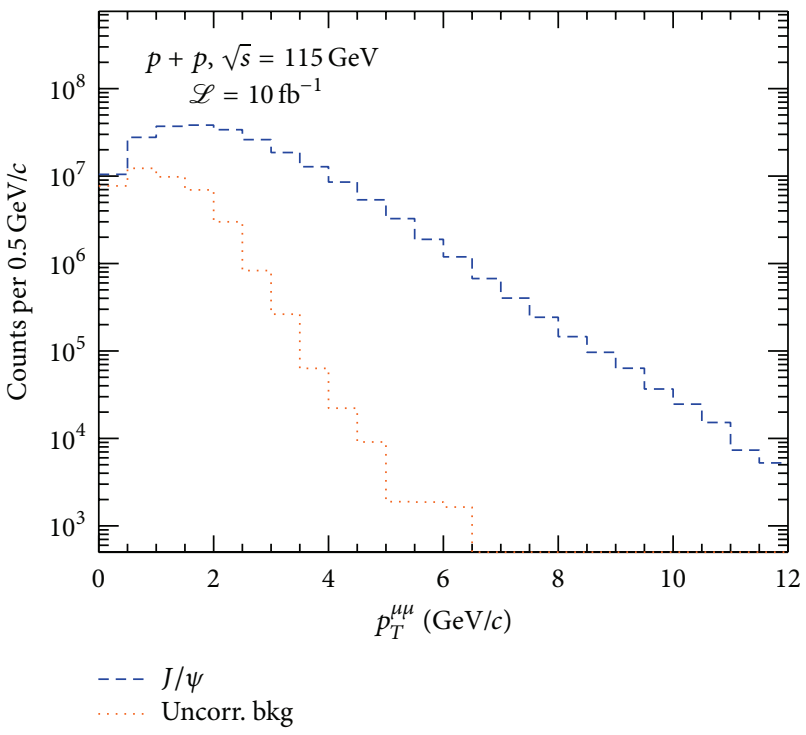

(a)

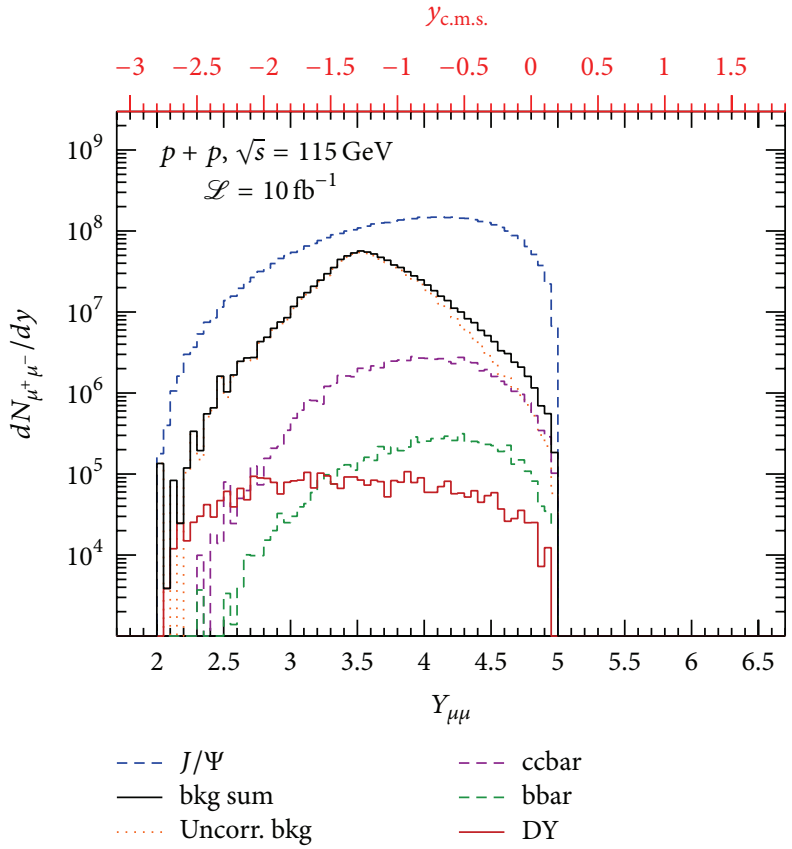

(b)

Figure 7: $p_{T}(\mathrm{a})$ and $y(\mathrm{~b})$ spectra of $J / \psi$ signal and different background sources in the $J / \psi$ mass range.

$\sqrt{s_{\mathrm{NN}}}=72 \mathrm{GeV}$ in fixed-target mode. The charged particle multiplicity is dominated by the $\pi$ multiplicity. By comparing these three distributions as a function of the pseudorapidity of the particle in the laboratory frame as shown in Figure 10, one can conclude that the charged particle multiplicity in a fixed-target mode never exceeds the one obtained in $p+\mathrm{Pb}$ collisions at $\sqrt{s_{\mathrm{NN}}}=5 \mathrm{TeV}$ in the collider mode in the full pseudorapidity range: a detector with the LHCb capabilities will be able to run in such conditions.

5.2. Prospects for the Measurements of the Nuclear Modification Factors for $J / \psi$ and $\Upsilon$ in $p+P b$ Collisions at $\sqrt{s_{N N}}=$ $115 \mathrm{GeV}$. To illustrate the potential offered by AFTER@LHC in $p+\mathrm{Pb}$ collisions at $\sqrt{s_{\mathrm{NN}}}=115 \mathrm{GeV}$, we have evaluated, in this section, the impact of the nuclear modification of the gluon densities in nucleons within large nucleus, generically referred to as gluon shadowing, and its uncertainty as encoded in the nuclear PDF set EPS09. For that, we have used the probabilistic Glauber Monte-Carlo framework, JIN $[43,44]$, which allows us to encode different mechanisms for the partonic production and to interface these production processes with different cold nuclear matter effects, such as the aforementioned shadowing, in order to get the production cross sections for proton-nucleus and nucleus-nucleus collisions. JIN also straightforwardly computes any nuclear modification factor for minimum bias collisions or in specific centrality classes. In the case of proton-nucleus $(p+A)$ collisions, it is the ratio of the yield per inelastic collision in $p+A$ collisions to the yield in $p p$ collisions at the same energy multiplied by the average number of binary collisions in a typical $p+p$ collision, $\left\langle N_{\text {coll }}\right\rangle$ :

$$
R_{p A}=\frac{d N_{p A}}{\left\langle N_{\text {coll }}\right\rangle d N_{p p}} .
$$

In the presence of a net nuclear effect, $R_{p A}$ is defined such that it differs from unity. In the simplest case of minimum bias collisions, one should have

$$
R_{p A}=\frac{d \sigma_{p A}}{A d \sigma_{p p}} .
$$

As in [45], we have used the central curve of EPS09 as well as four specific extreme curves (minimal/maximal shadowing, minimal/maximal EMC effect), which reproduce the envelope of the gluon nPDF uncertainty encoded in EPS09 LO [46].

In addition to the modification of the partonic densities, quarkonium production in $p+A$ collisions can be affected by other effects, for instance, by the nuclear absorption which depends much on the nature of the object traversing the nuclear medium. If the meson is already formed, it may be affected more than a smaller preresonant pair. To discuss such an effect, it is useful to introduce the concept of the formation time, $t_{f}$, based on the Heisenberg uncertainty principle and the time, in the rest frame of the meson, to discriminate between two $S$ states, for instance, the $J / \psi$ and the $\psi(2 S)$. In fact, one finds $[45,47]$ that such a time is similar for the charmonium and bottomonium states and is on the order 


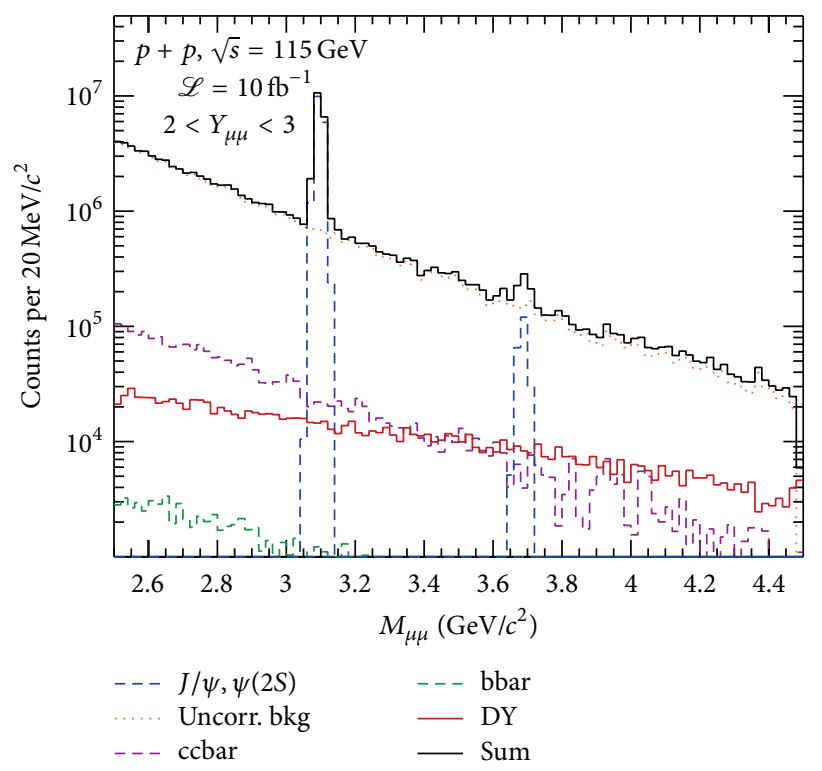

(a)

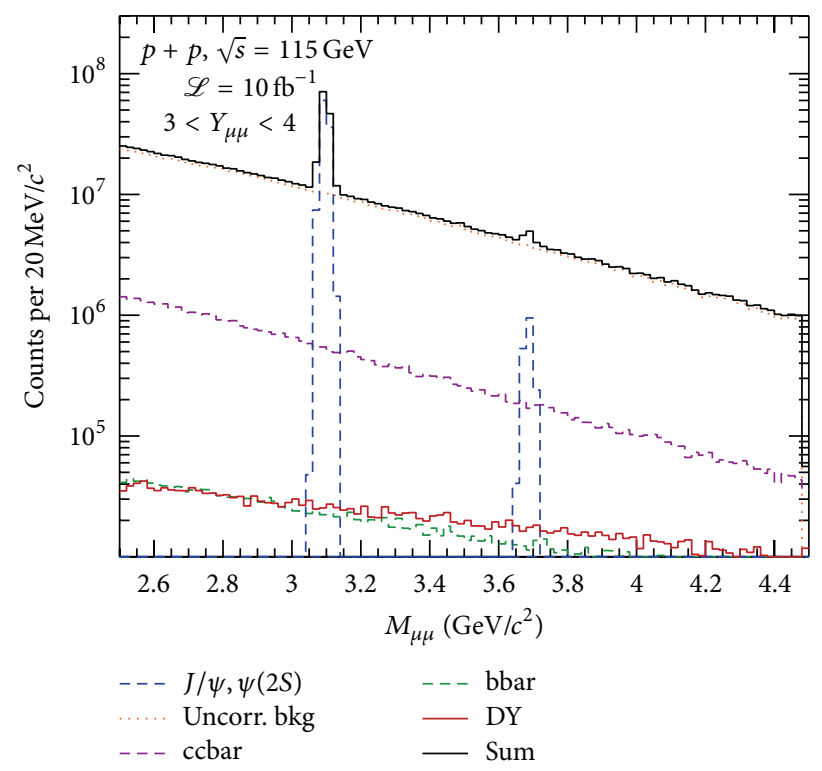

(b)

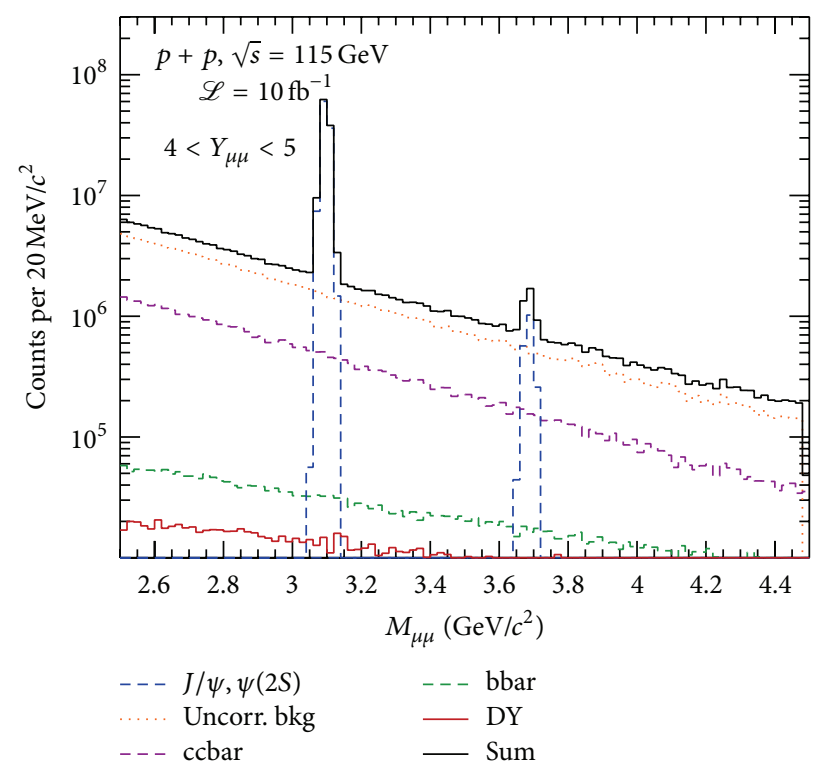

(c)

FIGURE 8: Dimuon invariant mass distributions for three rapidity bins in $J / \psi$ and $\psi(2 S)$ mass window. $2<Y_{\mu^{+} \mu^{-}}<3,3<Y_{\mu^{+} \mu^{-}}<4$, and $4<Y_{\mu^{+} \mu^{-}}<5$, shown on panels (a), (b), and (c), respectively.

of $0.3-0.4 \mathrm{fm}$. Obviously, this time has to be boosted in the frame where the nuclear matter sits. For $t_{f}$ smaller than the nucleus radius, the quarkonium is formed before escaping it. In the fixed-target mode with a proton beam and a nuclear target, the boost factor is simply $\gamma\left(y_{\text {lab }}\right)=\cosh \left(y_{\text {lab }}\right)$. We therefore obtain $t_{f}$ as in Table 4.

One sees that looking at quarkonium production in $p+\mathrm{Pb}$ collisions at different backward rapidities allows one to look at quarkonia traversing the nuclear matter at very different stages of their evolution. This effect could theoretically be studied by giving an ad hoc rapidity dependence to the effective absorption cross section, $\sigma_{\text {abs }}^{\text {effective }}$. This is left for
TABLE 4: Boost and formation time in the (target) $\mathrm{Pb}$ rest frame of $J / \psi$ and $\Upsilon$ as a function of its c.m.s. rapidity at $\sqrt{s_{\mathrm{NN}}}=115 \mathrm{GeV}$.

\begin{tabular}{cccccccc}
\hline$y_{\text {c.m.s }}$ & $y_{\text {lab }}$ & $\gamma\left(y_{\text {lab }}\right)$ & $t_{f}^{J / \psi, Y}(y)$ & $y_{\text {c.m.s }}$ & $y_{\text {lab }}$ & $\gamma\left(y_{\text {lab }}\right)$ & $t_{f}^{J / \psi, Y}(y)$ \\
\hline-2.5 & 2.3 & 5 & $1.75 \mathrm{fm}$ & -0.5 & 4.3 & 37 & $13 \mathrm{fm}$ \\
-1.5 & 3.3 & 14 & $5 \mathrm{fm}$ & 0.0 & 4.8 & 61 & $21 \mathrm{fm}$ \\
-1.0 & 3.8 & 22 & $8 \mathrm{fm}$ & 0.5 & 5.3 & 100 & $35 \mathrm{fm}$ \\
\hline
\end{tabular}

a future study since, here, we wish to consider only the nPDF effects and the expected statistics. Other effects to be considered are the coherent energy loss [48] (expected to 


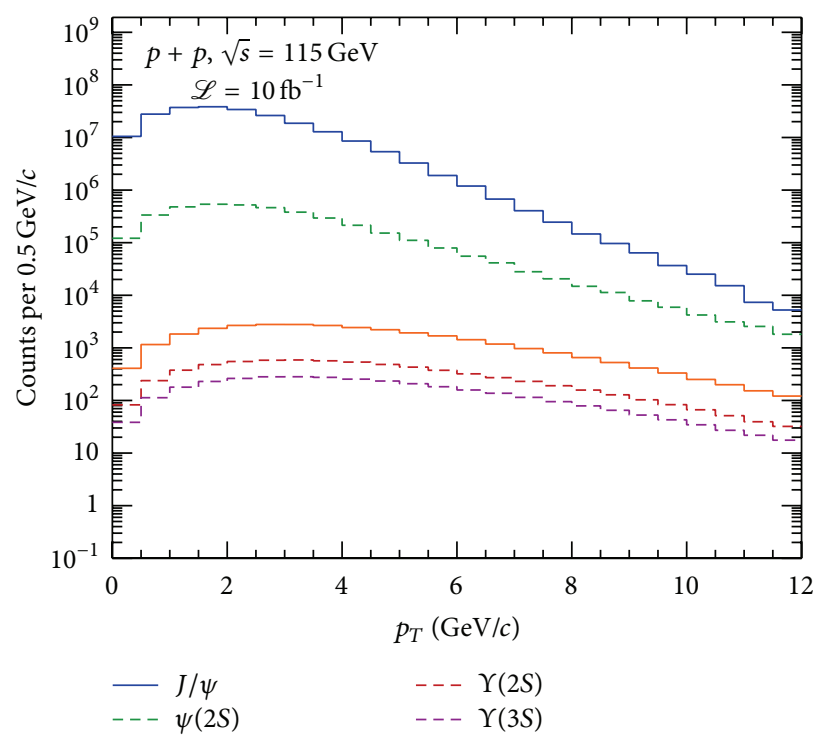

(a)

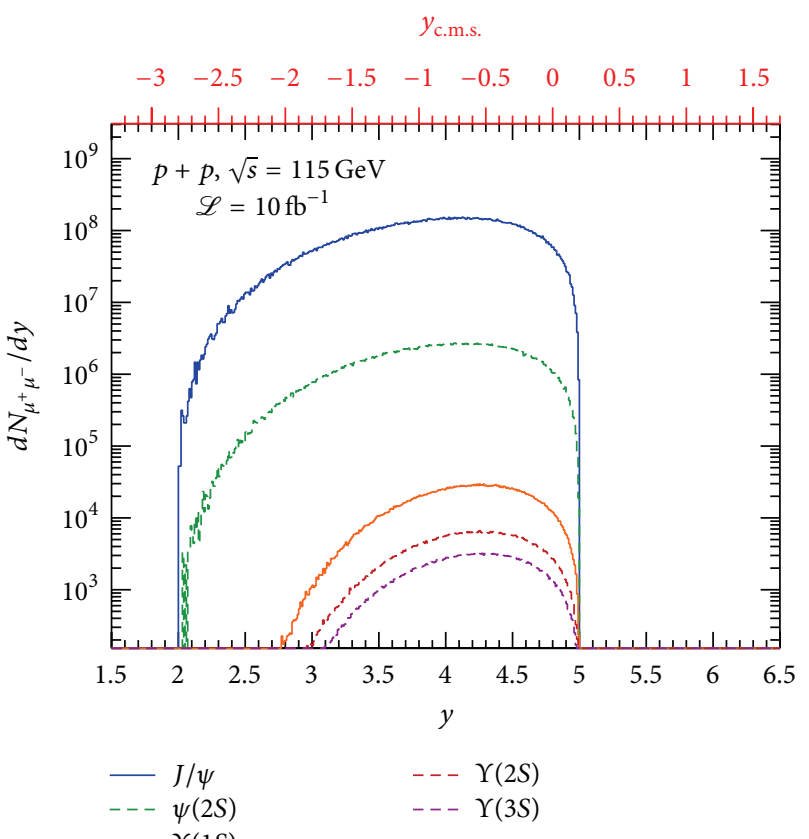

(b)

Figure 9: Transverse momentum (a) and rapidity (b) distributions for $J / \psi, \psi(2 S), \Upsilon(1 S), \Upsilon(2 S)$, and $\Upsilon(3 S)$ from the top to the bottom distribution.

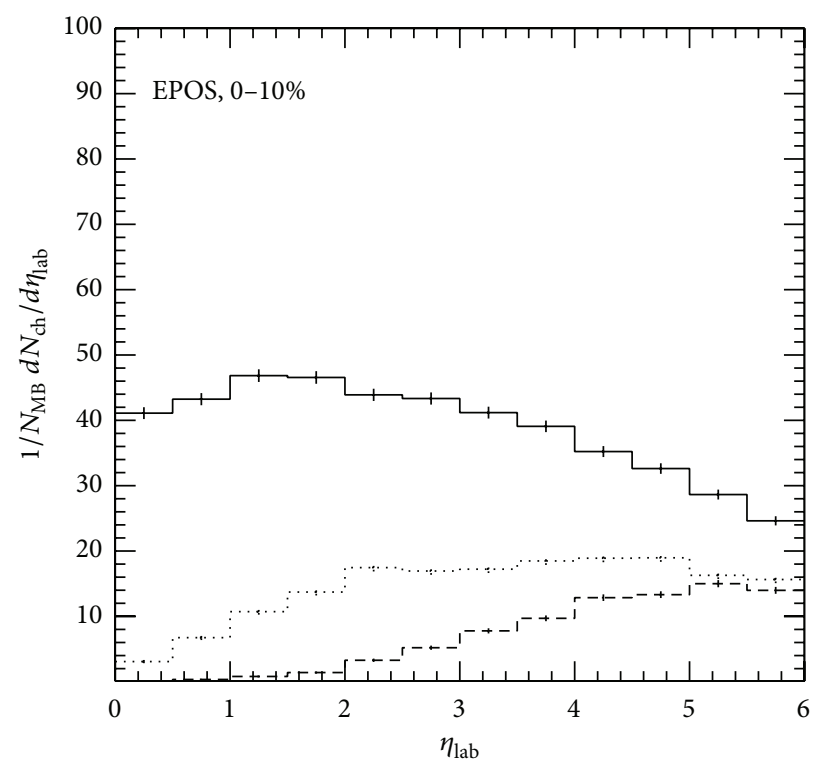

Fixed-target mode

- - Pb beam, $\mathrm{H}$ target, $\sqrt{s_{\mathrm{NN}}}=72 \mathrm{GeV}$

… $p$ beam, $\mathrm{Pb}$ target, $\sqrt{s_{\mathrm{NN}}}=115 \mathrm{GeV}$

Collider mode

- Pb beam, $p$ beam, $\sqrt{s_{\mathrm{NN}}}=5020 \mathrm{GeV}$

FIGURE 10: Averaged number of charged particles in $p+A$ collisions as a function of the pseudorapidity in the laboratory frame. 


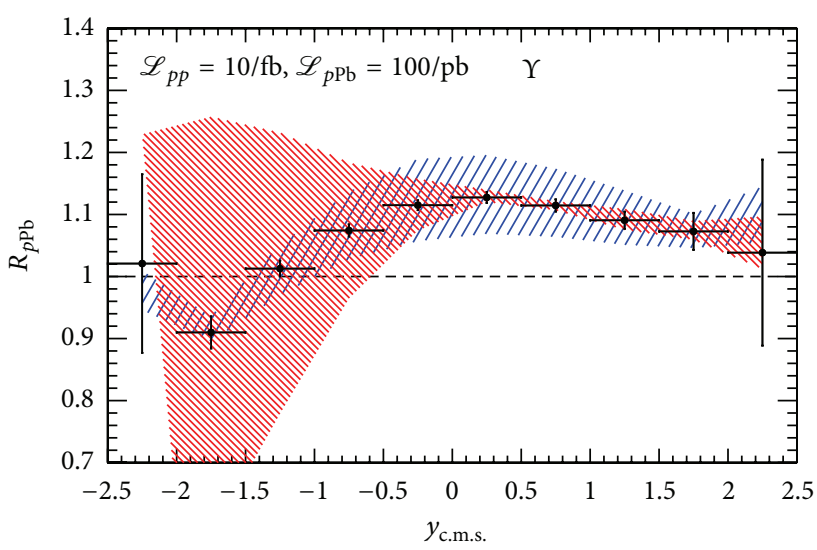

- EPS09 central

'HHH. EPS09 min./max. shadowing

तNH EPS09 min./max. EMC effect

(a)

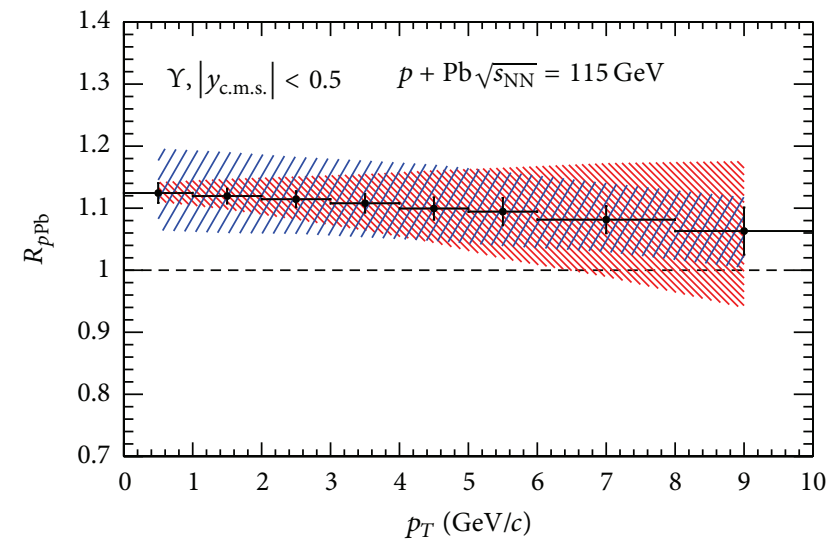

- EPS09 central

'HHH. EPS09 min./max. shadowing HWH EPS09 min./max. EMC effect

(b)

FIGURE 11: Nuclear modification factor for $\Upsilon$ as a function of (a) $y_{\mathrm{CMS}}$ and (b) $p_{T}$ in $p+\mathrm{Pb}$ collisions at $\sqrt{s_{\mathrm{NN}}}=115 \mathrm{GeV}$. The uncertainties attached to the central points are derived from the statistics to be collected with $\mathscr{L}_{p+p}=10 \mathrm{fb}^{-1}$ and $\mathscr{L}_{p+\mathrm{Pb}}=100 \mathrm{pb}^{-1}$.

grow in the forward region) and the rescattering by comovers [49] (expected to grow with the multiplicity along the $J / \psi$ direction).

Since we wish to assess the discriminating power of the possible data to be taken with AFTER@LHC, we attribute to the EPS09 central values statistical uncertainties which directly follow from the differential yields, respectively, expected in $p+p$ and $p+\mathrm{Pb}$ collisions. For that we take an integrated luminosity of $10 \mathrm{fb}^{-1}$ for the $p+p$ runs and $100 \mathrm{pb}^{-1}$ for the $p+\mathrm{Pb}$ runs, in accordance with the luminosities discussed above (see Table 1). As this stage, we do not consider additional systematical uncertainties. This simplifying assumption could be lifted in a more detailed study which would also take into account a possible detector acceptance (and related efficiencies) as done in the previous section. In particular, we do not expect that the rapidity region for $y_{\mathrm{CMS}}>1.5$ would be easily accessible.

In Figure 11, we show the rapidity dependence of $R_{p+\mathrm{Pb}}$ for $\Upsilon$ and its $p_{T}$ dependence near $y=0$. The only million of $\Upsilon$ to be collected per year allows for the measurement of a $R_{p+\mathrm{Pb}}$ with a much better precision than the gluon nPDF, nearly up to $x \rightarrow 1$. In addition, one notes that the nuclear modification factor is certainly measurable up to $p_{T} \simeq 10 \mathrm{GeV} / c$.

In Figure 12, we also show the rapidity dependence of $R_{p+\mathrm{Pb}}$ for $J / \psi$ and its $p_{T}$ dependence near $y=0$. In both cases, the luminosity to be taken in a year at AFTER@LHC yields to statistical uncertainties which are largely negligible as compared to the nPDF uncertainties; the statistical uncertainties are not even visible in Figure 12. We expect this to hold also for the $\psi(2 S)$ although its yields are down by a factor of 100 .

As aforementioned, the nPDFs do not account for all the expected nuclear matter effects. However, it is clear that combining the measurements of $\Upsilon, J / \psi$, and $\psi(2 S)$ for
$-3<y_{\mathrm{CMS}}<0$ (as a LHCb-like detector would do) will allow one to pin down the existence of a possible gluon EMC and antishadowing effect. We also stress that the complications induced by a rapidity dependence of $\sigma_{\text {abs }}^{\text {effective }}$ could be avoided by the parallel measurement of $R_{p+\mathrm{Pb}}$ for nonprompt $J / \psi$ which can only be sensitive to the energy loss since the $b$ quark decay (weakly) into the $J / \psi$, way outside the nucleus. Figure 13 shows that the trend is similar compared to $\Upsilon$. Measuring the $p_{T}$ dependence of $R_{p+\mathrm{Pb}}$ for prompt $J / \psi$ and $\Upsilon$ should also avoid the sensitivity on formation time effects.

\section{Prospects of $\mathbf{P b}+A$ Measurements at $\sqrt{s}=72 \mathbf{~ G e V}$}

The charged particle multiplicity has been generated with the EPOS generator $[41,42]$ in different configurations: $\mathrm{Pb}+\mathrm{Pb}$ at $\sqrt{s_{\mathrm{NN}}}=5.5 \mathrm{TeV}$ in collider mode, $\mathrm{Pb}+\mathrm{Ar}, \mathrm{Pb}+\mathrm{Xe}$, and $\mathrm{Pb}+\mathrm{Pb}$ at $\sqrt{s_{\mathrm{NN}}}=72 \mathrm{GeV}$ in fixed-target mode. By comparing these three distributions in the pseudorapidity of the particle in the laboratory frame as shown in Figure 14, one can conclude that the charged particle multiplicity in a fixed-target mode never exceeds the one obtained in $\mathrm{Pb}+\mathrm{Pb}$ collisions at $\sqrt{s_{\mathrm{NN}}}=5.5 \mathrm{TeV}$ obtained in a collider mode in the full pseudorapidity range: a detector with the ALICE MFT+Muon detector [50] capability will be able to run in such conditions. Detailed studies are needed to evaluate up to which multiplicity a detector such as LHCb would be able to take good quality data.

\section{Conclusion}

In summary, we have shown that in a fixed-target mode with an integrated luminosity of $10 \mathrm{fb}^{-1}$, using $7 \mathrm{TeV}$ LHC proton beam on a hydrogen target, and with a detector setup and performances similar to the LHCb detector, quarkonium 


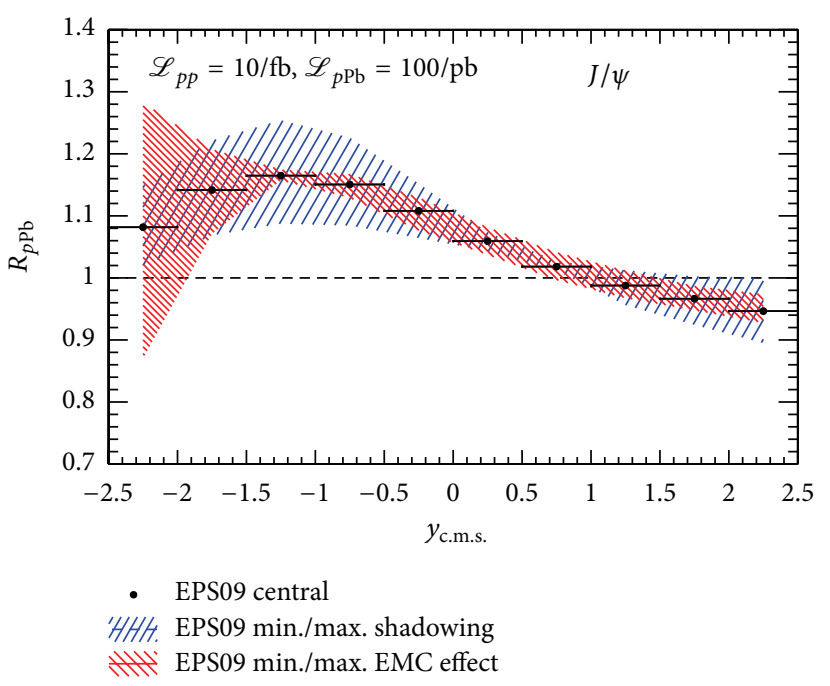

(a)

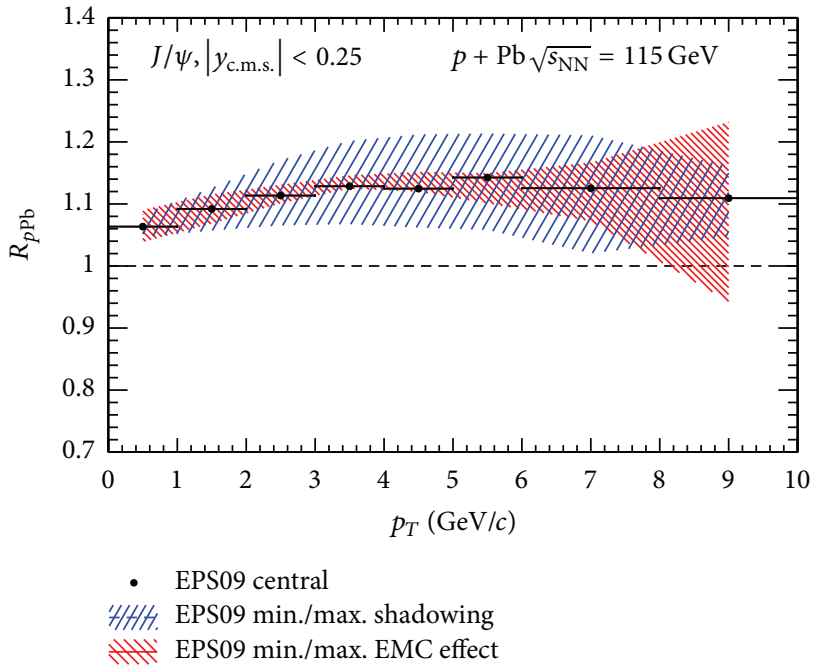

(b)

Figure 12: Nuclear modification factor for $J / \psi$ as a function of (a) $y_{\mathrm{CMS}}$ and (b) $p_{T}$ in $p+\mathrm{Pb}$ collisions at $\sqrt{s_{\mathrm{NN}}}=115 \mathrm{GeV}$. The uncertainties derived from the statistics to be collected with $\mathscr{L}_{p+p}=10 \mathrm{fb}^{-1}$ and $\mathscr{L}_{p+\mathrm{Pb}}=100 \mathrm{pb}^{-1}$ are smaller than the point size.

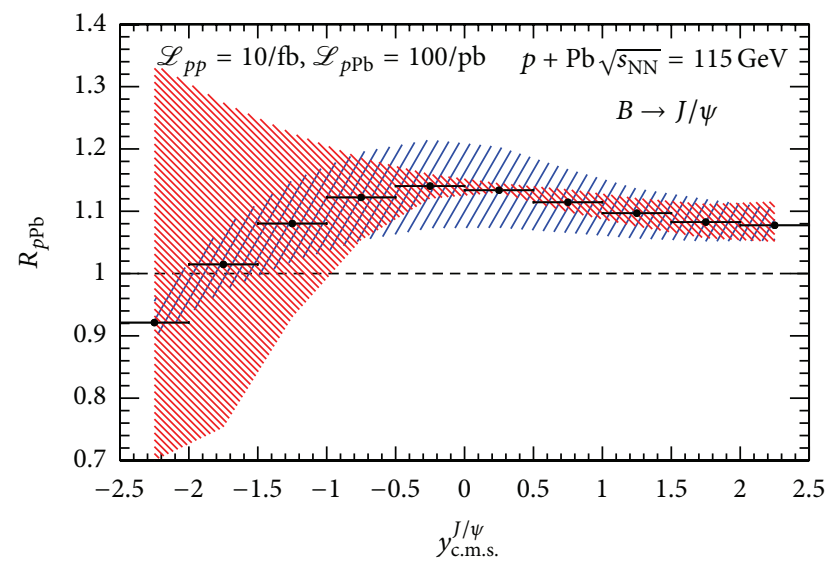

- EPS09 central

'HHF. EPS09 min./max. shadowing

ttt' EPS09 min./max. EMC effect

FIGURE 13: Nuclear modification factor for $J / \psi$ from $b$ as a function of $y_{\mathrm{CMS}}$ in $p+\mathrm{Pb}$ collisions at $\sqrt{{s_{\mathrm{NN}}}}=115 \mathrm{GeV}$. The uncertainties derived from the statistics to be collected with $\mathscr{L}_{p+p}=10 \mathrm{fb}^{-1}$ and $\mathscr{L}_{\mathrm{p}+\mathrm{Pb}}=100 \mathrm{pb}^{-1}$ are smaller than the point size.

studies in the dimuon decay channel can be performed over a wide transverse momentum range and rapidity in the center-of-mass from $\sim-2.8$ for $J / \psi$ and $\psi(2 S)$ and $\sim-2$ for $\Upsilon$ states to $\sim 0$. We have performed simulations of the dominant background sources contributing to the $\mu^{+} \mu^{-}$invariant mass spectrum. The uncorrelated background was obtained using Pythia generator and dimuons from correlated background sources, $c \bar{c}, b \bar{b}$, and Drell-Yan, were simulated using both HELAC-ONIA and PyTHIA generators. The estimated background level allows for $J / \psi, \psi(2 S), \Upsilon(1 S)$,

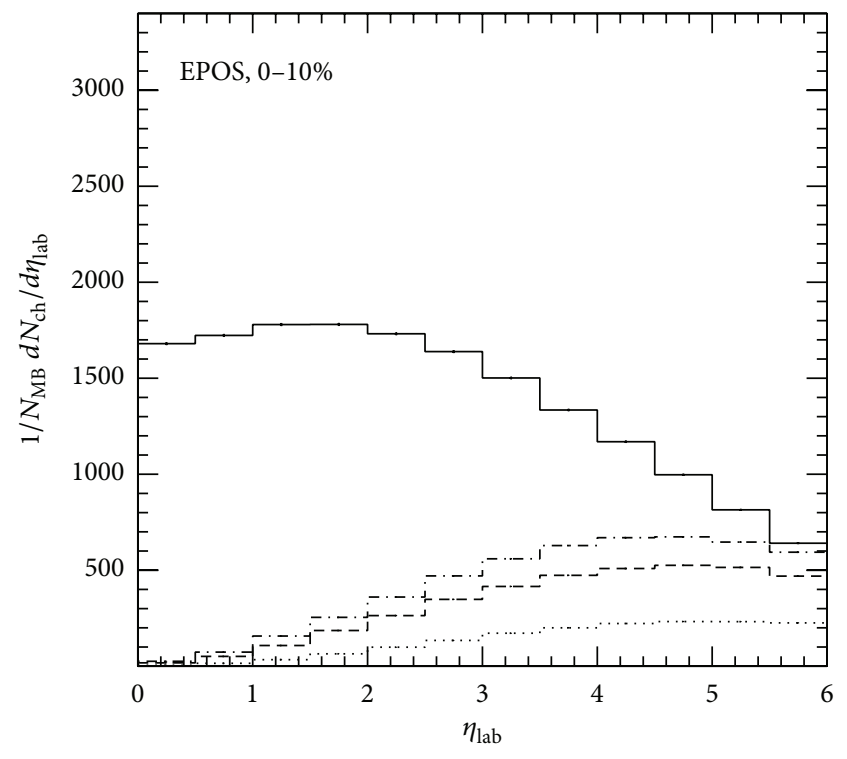

Fixed-target mode

-. - $\mathrm{Pb}$ beam, $\mathrm{Pb}$ target, $\sqrt{s_{\mathrm{NN}}}=72 \mathrm{GeV}$

- - - Pb beam, Xe target, $\sqrt{s_{\mathrm{NN}}}=72 \mathrm{GeV}$

..... Pb beam, Ar target, $\sqrt{s_{\mathrm{NN}}}=72 \mathrm{GeV}$

Collider mode

— Pb beam, $\mathrm{Pb}$ beam, $\sqrt{s_{\mathrm{NN}}}=5500 \mathrm{GeV}$

FIgURE 14: Averaged number of charged particles in $A+A$ collisions as a function of the pseudorapidity in the laboratory frame.

$\Upsilon(2 S)$, and $\Upsilon(3 S)$ measurements in the dimuon decay channel with good signal to background ratios.

These simulations set the stage for further ones including, on the one hand, the detection of photon from $P$ wave or $\eta_{c}$ decay or from the production of a $J / \psi+\gamma$ pair, whose studies 
at low transverse momentum can provide important insight on the gluon transverse dynamics [51-54], and, on the other hand, the large combinatorial background typical of $p+A$ and $A+A$ collisions in which the study of excited quarkonium at AFTER@LHC energies is of paramount importance $[1,13]$. We note that the Delphes [55] framework seems particularly well suited to account for the photon detectability in such prospective studies.

Along our investigations, we have also noted that the main source of dimuons around the $\Upsilon(n S)$ masses is from the Drell-Yan process (see Figure 6(b)). This gives us great confidence that the corresponding cross section can easily be extracted in this mass region in $p+p$ collisions, a fortiori with a vertex detector allowing for tagging the heavy-flavour muons. We therefore consider that the single-spin asymmetries for Drell-Yan pair production can indeed be extracted using a light polarised target. Motivations for such studies are discussed in $[15,16,56]$. Quarkonium polarisation measurements are of course also possible, given the large statistical samples.

As regards the case of $p+A$ collisions, we have had a first look at the charged particle multiplicities as a function of the laboratory pseudorapidity. We have found out that, for all the possible fixed-target modes, $p+\mathrm{Pb}, \mathrm{Pb}+\mathrm{H}$, and $\mathrm{Pb}+\mathrm{Pb}$, these are smaller than the ones reached in the collider modes, where the $\mathrm{LHCb}$ was used $(p+\mathrm{Pb}$ and $\mathrm{Pb}+p$ at $5 \mathrm{TeV})$. We therefore believe that a detector with similar characteristics as compared to LHCb can very well be used in the fixed-target mode (our observation is obviously supported by the preliminary analysis of the LHCb-SMOG data taken during the pilot run of $p^{+}$beam $(\mathrm{Pb}$ beam) on a Neon gas target from 2012 (2013) at a c.m.s energy of $\sqrt{s_{\mathrm{NN}}}=87 \mathrm{GeV}$ (54 GeV) [57]).

In view of the above, we have evaluated the impact, and its uncertainty, on the nuclear modification of the gluon densities on prompt and nonprompt $J / \psi$ and $\Upsilon$ in the form of $R_{p+\mathrm{Pb}}$. We have found that the measurements at backward rapidities allow one to search for the gluon antishadowing, the gluon EMC effect, and even the Fermi motion effect on the gluons with the unheard statistical precisions. The statistics are large enough to perform such measurement with the $\psi(2 S)$ and probably also with $\Upsilon(2 S)$ and $\Upsilon(3 S)$ allowing for thorough investigations of the formation time effect of the meson propagating in the nuclear matter. Overall, our results confirm the great potential of AFTER@LHC for heavy-quark and quarkonium physics.

\section{Conflict of Interests}

The authors declare that there is no conflict of interests regarding the publication of this paper.

\section{Acknowledgments}

The authors thank C. Da Silva, D. d'Enterria, E. G. Ferreiro, R. Mikkelsen, S. Porteboeuf-Houssais, A. Rakotozafindrabe, P. Robbe, M. Selvaggi, M. Schmelling, P. Skands, and Z. Yang for the important and stimulating discussions. This research was supported in part by the ERC Grant 291377
"LHCtheory: Theoretical predictions and analyses of LHC physics: advancing the precision frontier," by the COPININ2P3 Agreement, by the French P2I0 Excellence Laboratory, by the French CNRS via the grants PICS-06149 Torino-IPNO and FCPPL-Quarkonium4AFTER \& PEPS4AFTER2, by the European Social Fund within the framework of realizing the project "Support of intersectoral mobility and quality enhancement of research teams at Czech Technical University in Prague," CZ.1.07/2.3.00/30.0034, by Grant Agency of the Czech Republic, Grant no. 13-20841S, and by the Foundation for Polish Science Grant HOMING PLUS/2013-7/8.

\section{References}

[1] S. J. Brodsky, F. Fleuret, C. Hadjidakis, and J. P. Lansberg, "Physics opportunities of a fixed-target experiment using LHC beams," Physics Reports, vol. 522, no. 4, pp. 239-255, 2013.

[2] G. Arduini, K. Elsener, G. Fidecaro et al., "On the energy dependence of proton beam extraction with a bent crystal," Physics Letters B, vol. 422, no. 1-4, pp. 325-333, 1998.

[3] W. Scandale, G. Arduini, R. Assmann et al., "Comparative results on collimation of the SPS beam of protons and $\mathrm{Pb}$ ions with bent crystals," Physics Letters B, vol. 703, no. 5, pp. 547-551, 2011.

[4] E. Uggerhoj and U. I. Uggerhoj, "Strong crystalline fields: a possibility for extraction from the LHC," Nuclear Instruments and Methods in Physics Research B, vol. 234, pp. 31-39, 2005.

[5] LHC Committee, "Minutes of 107th meeting," Tech. Rep. CERN/LHC, 2011.

[6] C. Barschel, Precision luminosity measurement at $\mathrm{LHCb}$ with beam-gas imaging [Ph.D. thesis], RWTH Aachen University, 2013.

[7] M. Ferro-Luzzi, "Proposal for an absolute luminosity determination in colliding beam experiments using vertex detection of beam-gas interactions," Nuclear Instruments and Methods in Physics Research A, vol. 553, no. 3, pp. 388-399, 2005.

[8] R. Aaij, B. Adeva, M. Adinolfi et al., "Precision luminosity measurements at LHCb," Journal of Instrumentation, vol. 9, no. 12, Article ID P12005, 2014.

[9] A. Adare, S. Afanasiev, C. Aidala et al., "Ground and excited state charmonium production in $p+p$ collisions at $\sqrt{s}=200$ GeV," Physical Review D, vol. 85, Article ID 092004, 2012.

[10] R. Aaij, C. Abellan Beteta, B. Adeva et al., "Measurement of $\Upsilon$ production in $p p$ collisions at $\sqrt{s}=7 \mathrm{TeV}$," The European Physical Journal C, vol. 72, article 2025, 2012.

[11] A. Rakotozafindrabe, M. Anselmino, R. Arnaldi et al., "Spin physics at a fixed-target experiment at the LHC (AFTER@LHC)," Physics of Particles and Nuclei, vol. 45, no. 1, pp. 336-337, 2014.

[12] N. Brambilla, S. Eidelman, B. K. Heltsley et al., "Heavy quarkonium: progress, puzzles, and opportunities," The European Physical Journal C, vol. 71, article 1534, 2011.

[13] J. P. Lansberg, S. J. Brodsky, F. Fleuret, and C. Hadjidakis, "Quarkonium physics at a fixed-target experiment using the LHC beams," Few-Body Systems, vol. 53, no. 1-2, pp. 11-25, 2012.

[14] J. P. Lansberg, R. Arnaldi, S. J. Brodsky et al., "AFTER@LHC: a precision machine to study the interface between particle and nuclear physics," EPJ Web of Conferences, vol. 66, Article ID $11023,2014$.

[15] T. Liu and B.-Q. Ma, "Azimuthal asymmetries in lepton-pair production at a fixed-target experiment using the LHC beams 
(AFTER)," The European Physical Journal C, vol. 72, article 2037, 2012.

[16] K. Kanazawa, Y. Koike, A. Metz, and D. Pitonyak, "Transverse single-spin asymmetries in proton-proton collisions at the AFTER@LHC experiment," Advances in High Energy Physics. Accepted.

[17] A. Rakotozafindrabe, R. Arnaldi, S. J. Brodsky et al., "Ultrarelativistic heavy-ion physics with AFTER@LHC," Nuclear Physics A, vol. 904-905, pp. 957c-960c, 2013.

[18] H.-S. Shao, "HELAC-Onia: an automatic matrix element generator for heavy quarkonium physics," Computer Physics Communications, vol. 184, no. 11, pp. 2562-2570, 2013.

[19] J. Alwall, A. Ballestrero, P. Bartalini et al., "A standard format for Les Houches event files," Computer Physics Communications, vol. 176, no. 4, pp. 300-304, 2007.

[20] T. Sjöstrand, S. Mrenna, and P. Skands, "A brief introduction to PYTHIA 8.1," Computer Physics Communications, vol. 178, no. 11, pp. 852-867, 2008.

[21] C. H. Kom, A. Kulesza, and W. J. Stirling, "Pair production of $\mathrm{J} /$ psi as a probe of double parton scattering at LHCb," Physical Review Letters, vol. 107, no. 8, Article ID 082002, 2011.

[22] K. Olive, K. Agashe, C. Amsler et al., "Review of particle physics," Chinese Physics C, vol. 38, Article ID 090001, 2014.

[23] A. D. Martin, W. J. Stirling, R. S. Thorne, and G. Watt, "Parton distributions for the LHC," The European Physical Journal C, vol. 63, no. 2, pp. 189-285, 2009.

[24] M. Whalley, D. Bourilkov, and R. Group, "The les Houches accord PDFs (LHAPDF) and LHAGLUE," http://arxiv.org/abs/ hep-ph/0508110.

[25] D. Acosta, T. Affolder, H. Akimoto et al., " $\Upsilon$ production and polarization in $p \bar{p}$ collisions at $\sqrt{s}=1.8 \mathrm{TeV}$," Physical Review Letters, vol. 88, Article ID 161802, 2002.

[26] G. Aad, B. Abbott, J. Abdallah et al., "Measurement of upsilon production in $7 \mathrm{TeV}$ pp collisions at ATLAS," Physical Review D, vol. 87, no. 5, Article ID 052004, 2013.

[27] S. Chatrchyan, V. Khachatryan, A. M. Sirunyan et al., "Measurement of the $\Upsilon(1 S), \Upsilon(2 S)$, and $\Upsilon(3 S)$ cross sections in $p p$ collisions at $\sqrt{s}=7 \mathrm{TeV}$,' Physics Letters B, vol. 727, no. 1-3, pp. 101-125, 2013.

[28] R. Aaij, C. Abellan Beteta, B. Adeva et al., "Production of $J / \psi$ and $\Upsilon$ mesons in $p p$ collisions at $\sqrt{s}=8 \mathrm{TeV}$," Journal of High Energy Physics, vol. 2013, no. 6, article 64, 2013.

[29] STAR Collaboration and Z. Ye, "Open charm hadron production in $p+p, \mathrm{Au}+\mathrm{Au}$ and $\mathrm{U}+\mathrm{U}$ collisions at STAR," Nuclear Physics A, vol. 931, pp. 520-524, 2014.

[30] J. Pumplin, D. R. Stump, J. Huston, H.-L. Lai, P. Nadolsky, and W.-K. Tung, "New generation of parton distributions with uncertainties from global QCD analysis," Journal of High Energy Physics, vol. 6, no. 7, pp. 325-371, 2002.

[31] J. Alwall, R. Frederix, S. Frixione et al., "The automated computation of tree-level and next-to-leading order differential cross sections, and their matching to parton shower simulations," Journal of High Energy Physics, vol. 2014, no. 7, article 079, 2014.

[32] J. C. Webb, T. C. Awes, M. L. Brooks et al., "Absolute DrellYan dimuon cross sections in $800 \mathrm{GeV} / \mathrm{c}$ pp and pd collisions," http://arxiv.org/abs/hep-ex/0302019.

[33] C. Anastasiou, L. J. Dixon, K. Melnikov, and F. Petriello, "Dilepton rapidity distribution in the Drell-Yan process at NNLO in QCD," Physical Review Letters, vol. 91, Article ID 182002, 2003.
[34] A. Buckley, J. Ferrando, S. Lloyd et al., "LHAPDF6: parton density access in the LHC precision era," The European Physical Journal C, vol. 75, no. 3, p. 132, 2015.

[35] A. Augusto Alves Jr., l. Andrade Filho, A. F. Barbosa et al., "The LHCb detector at the LHC," Journal of Instrumentation, vol. 3, Article ID S08005, 2008.

[36] R. Aaij, B. Adeva, M. Adinolfi et al., "Measurement of $J / \Psi$ production in $p p$ collisions at $\sqrt{s}=7 \mathrm{TeV}$," The European Physical Journal C, vol. 71, article 1645, 2011.

[37] F. Archilli, W. Baldini, G. Bencivenni et al., "Performance of the Muon identification at LHCb," Journal of Instrumentation, vol. 8, Article ID P10020, 2013.

[38] R. Aaij, B. Adeva, M. Adinolfi et al., "LHCb detector performance," International Journal of Modern Physics A, vol. 30, no. 7, Article ID 1530022, 2015.

[39] R. Aaij, B. Adeva, M. Adinolfi et al., "Study of $J / \psi$ production and cold nuclear matter effects in $p \mathrm{~Pb}$ collisions at $\sqrt{s_{N N}}=5$ TeV," Journal of High Energy Physics, vol. 2014, no. 2, article 72, 2014.

[40] R. Aaij, B. Adeva, M. Adinolfi et al., "Study of $\Upsilon$ production and cold nuclear matter effects in $p \mathrm{~Pb}$ collisions at $\sqrt{s_{N N}}=5 \mathrm{TeV}$," Journal of High Energy Physics, vol. 2014, no. 7, article 094, 2014.

[41] T. Pierog, I. Karpenko, J. Katzy, E. Yatsenko, and K. Werner, "EPOS LHC: test of collective hadronization with LHC data," http://arxiv.org/abs/1306.0121.

[42] K. Werner, F.-M. Liu, and T. Pierog, "Parton ladder splitting and the rapidity dependence of transverse momentum spectra in deuteron-gold collisions at the BNL Relativistic Heavy Ion Collider," Physical Review C, vol. 74, no. 4, Article ID 044902, 11 pages, 2006.

[43] E. Ferreiro, F. Fleuret, and A. Rakotozafindrabe, "Transversemomentum dependence of $J / \psi$ shadowing effects," The European Physical Journal C, vol. 61, no. 4, pp. 859-864, 2009.

[44] E. G. Ferreiro, F. Fleuret, J. P. Lansberg, and A. Rakotozafindrabe, "Cold nuclear matter effects on $\mathrm{J} / \psi$ production: intrinsic and extrinsic transverse momentum effects," Physics Letters B, vol. 680, no. 1, pp. 50-55, 2009.

[45] E. G. Ferreiro, F. Fleuret, J. P. Lansberg, and A. Rakotozafindrabe, "Impact of the nuclear modification of the gluon densities on $J / \psi$ production in $p \mathrm{~Pb}$ collisions at $\sqrt{s_{N N}}=5 \mathrm{TeV}$," Physical Review C, vol. 88, no. 4, Article ID 047901, 2013.

[46] K. Eskola, H. Paukkunen, and C. Salgado, "EPS09: a new generation of NLO and LO nuclear parton distribution functions," Journal of High Energy Physics, vol. 2009, no. 4, article 065, 2009.

[47] E. G. Ferreiro, F. Fleuret, J. P. Lansberg, N. Matagne, and A. Rakotozafindrabe, " $\Upsilon$ production in $p(d) A$ collisions at RHIC and the LHC," The European Physical Journal C, vol. 73, article 2427, 2013.

[48] F. Arleo and S. Peigné, " $J / \psi$ suppression in p-A collisions from parton energy loss in cold QCD matter," Physical Review Letters, vol. 109, no. 12, Article ID 122301, 2012.

[49] E. Ferreiro, " $\psi(2 S)$ versus $J / \psi$ suppression in the comover interaction approach," http://arxiv.org/abs/1411.0549.

[50] ALICE Collaboration, "Addendum of the letter of intent for the upgrade of the ALICE experiment: the Muon forward tracker," Tech. Rep. CERN-LHCC-2013-014. LHCC-I-022ADD-1, CERN, Geneva, Switzerland, 2013, https://cds.cern.ch/ record/1592659.

[51] D. Boer and C. Pisano, "Polarized gluon studies with charmonium and bottomonium at LHCb and after," Physical Review D-Particles, Fields, Gravitation and Cosmology, vol. 86, no. 9, Article ID 094007, 2012. 
[52] J. P. Ma, J. X. Wang, and S. Zhao, "Transverse momentum dependent factorization for quarkonium production at low transverse momentum," Physical Review D, vol. 88, no. 1, Article ID 014027, 2013.

[53] W. J. den Dunnen, J.-P. Lansberg, C. Pisano, and M. Schlegel, "Accessing the transverse dynamics and polarization of gluons inside the proton at the LHC," Physical Review Letters, vol. 112, no. 21, Article ID 212001, 2014.

[54] J. P. Lansberg, "Back-to-back isolated photon-quarkonium production at the LHC and the transverse-momentum-dependent distributions of the gluons in the proton," http://arxiv.org/abs/ 1502.02263 .

[55] J. de Favereau, C. Delaere, P. Demin et al., "DELPHES 3: a modular framework for fast simulation of a generic collider experiment," Journal of High Energy Physics, vol. 2014, no. 2, article 057, 2014.

[56] M. Anselmino, U. D'Alesio, and S. Melis, "Transverse single-spin asymmetries in proton-proton collisions at the AFTER@LHC experiment in a TMD factorisation scheme," http://arxiv.org/abs/1504.03791.

[57] R. Aaij, J. de Miranda, A. Gomes et al., "First analysis of the $p \mathrm{~Pb}$ pilot run data with LHCb," LHCb-CONF-2012-034, January 2013. 

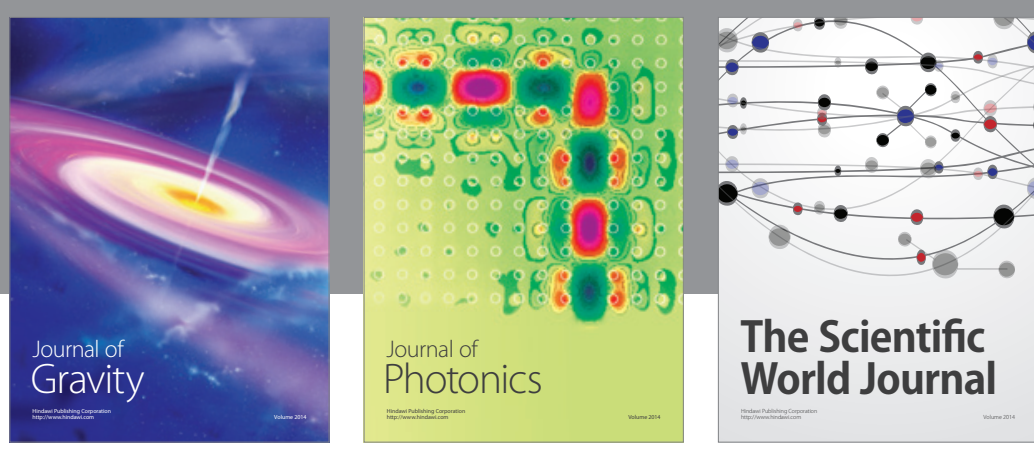

The Scientific World Journal
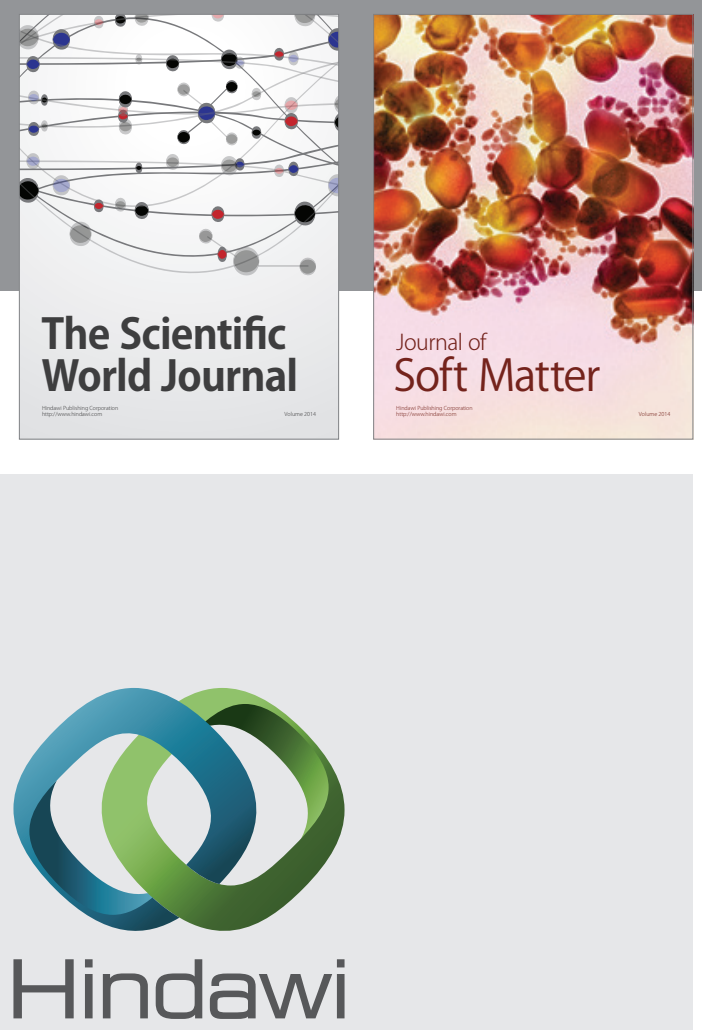

Submit your manuscripts at

http://www.hindawi.com

nternational Journal of

Statistical Mechanics
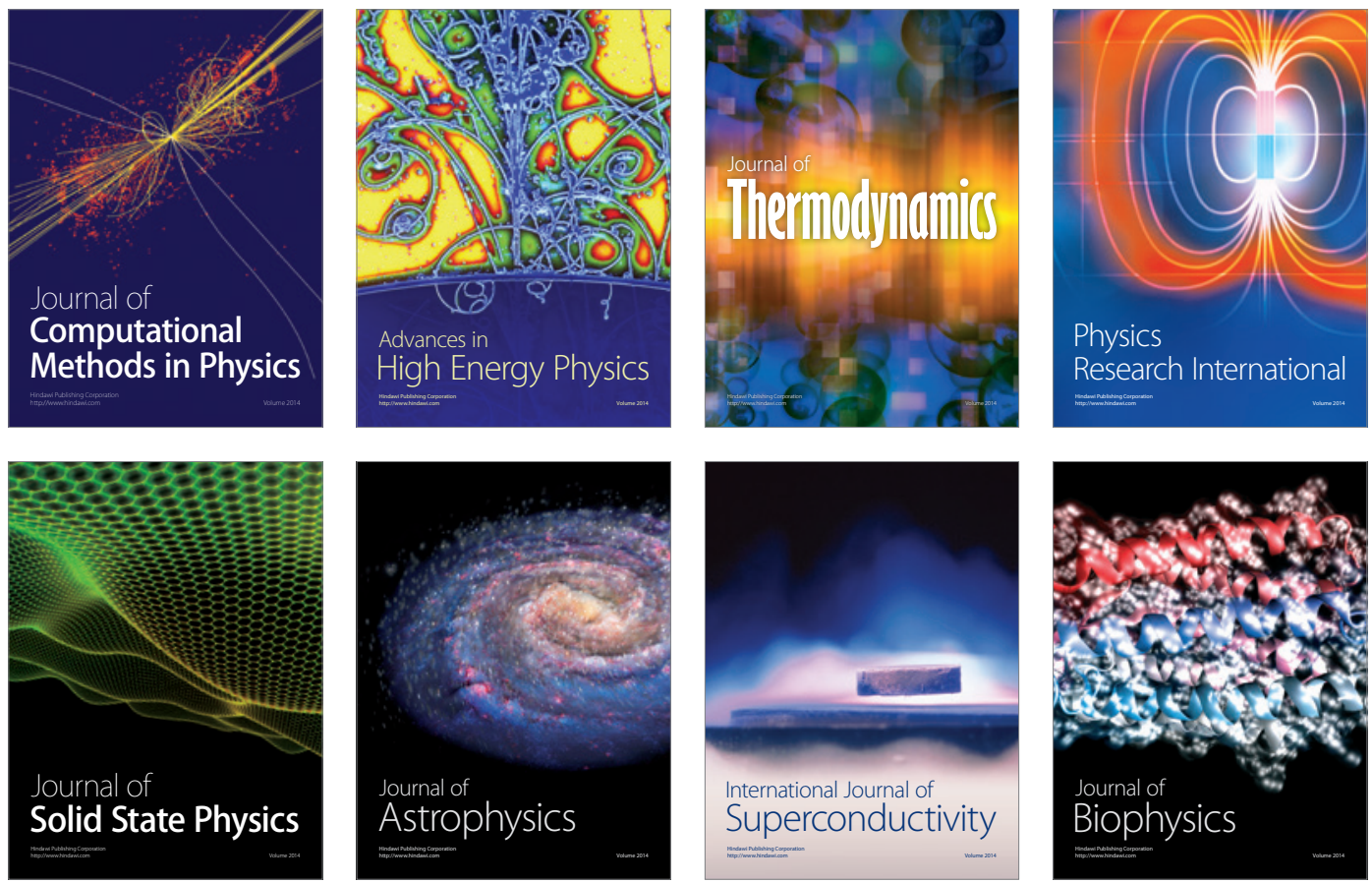
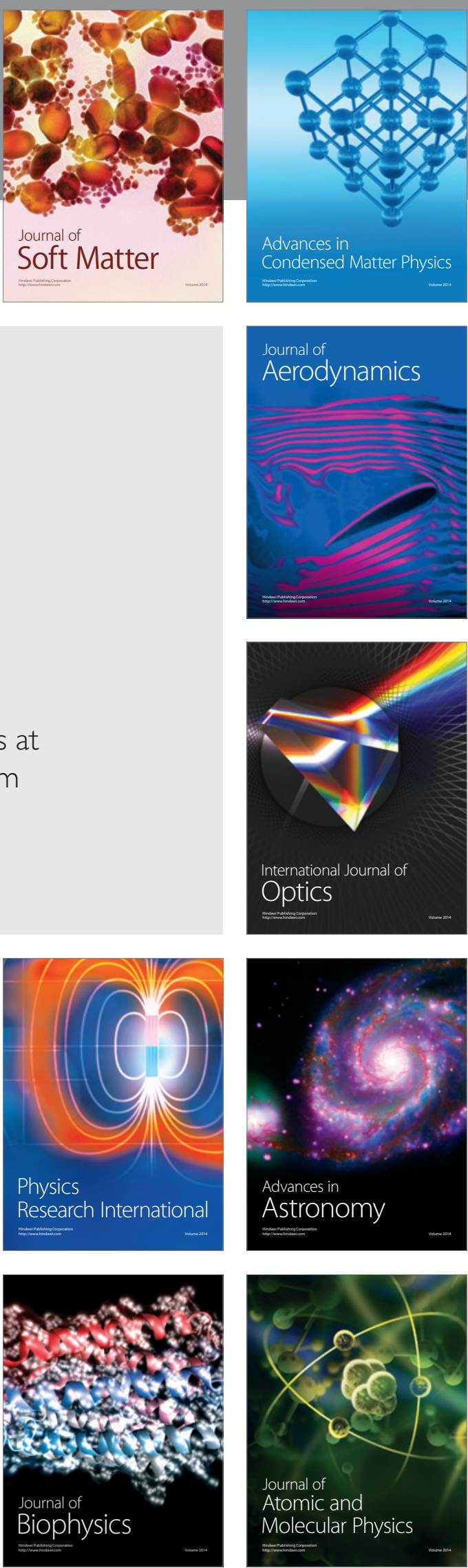